\title{
Existence of Weak Solutions for a Diffuse Interface Model for Viscous, Incompressible Fluids with General Densities
}

\section{Helmut Abels}

Max Planck Institute for Mathematics in Science, Inselstr. 22, 04103 Leipzig, Germany. E-mail: abels@mis.mpg.de

Received: 21 January 2008 / Accepted: 23 February 2009

Published online: 21 April 2009 - (C) The Author(s) 2009. This article is published with open access at Springerlink.com

\begin{abstract}
We study a diffuse interface model for the flow of two viscous incompressible Newtonian fluids in a bounded domain. The fluids are assumed to be macroscopically immiscible, but a partial mixing in a small interfacial region is assumed in the model. Moreover, diffusion of both components is taken into account. In contrast to previous works, we study the general case that the fluids have different densities. This leads to an inhomogeneous Navier-Stokes system coupled to a Cahn-Hilliard system, where the density of the mixture depends on the concentration, the velocity field is no longer divergence free, and the pressure enters the equation for the chemical potential. We prove existence of weak solutions for the non-stationary system in two and three space dimensions.
\end{abstract}

\section{Introduction and Main Result}

In this article we consider a so-called diffuse interface model for two viscous, incompressible Newtonian fluids of different density. In the model a partial mixing of the macroscopically immiscible fluids is considered and diffusion effects are taken into account. Such models have been successfully used to describe flows of two or more fluids macroscopically beyond the occurrence of topological singularities of the separating interface (e.g. coalescence or formation of drops). We refer to Anderson and McFadden [5] for a review on that topic.

The model which we are considering leads (after a reduction) to the system

$$
\begin{gathered}
\rho \partial_{t} v+\rho v \cdot \nabla v-\operatorname{div} S(c, D v)+\rho \nabla g_{0}=\rho \mu_{0} \nabla c \text { in } Q, \\
\partial_{t} \rho+\operatorname{div}(\rho v)=0 \text { in } Q, \\
\rho \partial_{t} c+\rho v \cdot \nabla c=\operatorname{div}\left(m(c) \nabla \mu_{0}\right) \text { in } Q, \\
\rho \mu_{0}+\rho^{2} \bar{p}=\beta \rho^{2} g_{0}-a^{\frac{1}{q}}(c) \Delta_{q} A(c)+\phi(c) \text { in } Q,
\end{gathered}
$$


together with

$$
\int_{\Omega} \mu_{0}(t) d x=\int_{\Omega} g_{0}(t) d x=0, \quad t \in(0, \infty)
$$

where $Q=\Omega \times(0, \infty)$ and $\Omega \subset \mathbb{R}^{d}, d=2,3$, is a bounded domain with $C^{2}$-boundary. Here $v$ and $\rho=\hat{\rho}(c)$ are the (mean) velocity and the density of the mixture of the two fluids, $g_{0}$ is a modified pressure, $c$ is the concentration difference of the two fluids, $\mu$ is the chemical potential associated to $c, \mu_{0}$ is the mean-value free part of $\mu$, and $\bar{p}$ is a constant (depending on time), which is related to the mean values of the pressure and the chemical potential. Moreover,

$$
S(c, D v)=2 v(c) D v+\eta(c) \operatorname{div} v I, \quad \hat{\Lambda}^{\prime}(s)=a^{\frac{1}{q}}(s), \hat{\Lambda}(0)=0,
$$

where $S(c, D v)$ is the stress tensor, $D v=\frac{1}{2}\left(\nabla v+\nabla v^{T}\right), v(c), \eta(c)>0$ are two viscosity coefficients, $\Delta_{q} u=\operatorname{div}\left(|\nabla u|^{q-2} \nabla u\right)$ is the $q$-Laplacian, $\nabla A \otimes \nabla A=\left(\partial_{j} A \partial_{k} A\right)_{j, k=1}^{d}$, $A=\hat{A}(c)$ is an auxiliary function related to $a(c)>0$, which arises in the free energy density of the system, and $m(c)>0$ is a mobility coefficient. Furthermore, $\Phi(c)$ is the homogeneous free energy density for the mixture and $\phi(c)=\Phi^{\prime}(c)$. Finally, we assume that the fluids behave like a simple mixture, cf. Lowengrub and Truskinovski [13], which means that the excess volume of the mixture is zero. This implies that $\hat{\rho}(c)=\frac{1}{\alpha+\beta c}$ for some $\alpha>0$ and $|\beta|<\alpha$.

We close the system by adding the boundary and initial conditions

$$
\begin{gathered}
\left.n \cdot v\right|_{\partial \Omega}=n \cdot S(c, D v)_{\tau}+\left.\gamma v_{\tau}\right|_{\partial \Omega}=0 \text { on } S, \\
\left.\partial_{n} c\right|_{\partial \Omega}=\left.\partial_{n} \mu_{0}\right|_{\partial \Omega}=0 \text { on } S, \\
\left.(v, c)\right|_{t=0}=\left(v_{0}, c_{0}\right) \text { in } \Omega,
\end{gathered}
$$

where $S=\partial \Omega \times(0, \infty), n$ is the exterior normal on $\partial \Omega$ and $0<\gamma<\infty$ is a friction coefficient.

In the case $a(c)=\hat{\rho}(c), q=2$ the model was proposed by Lowengrub and Truskinovski [13] as a generalization of a well-known diffuse interface model in the case of matched densities which corresponds to the case $\beta=0, \hat{\rho}(c) \equiv$ const., respectively, cf. e.g. Gurtin et al. [10]. The present modification can be derived in the same way, cf. e.g. [2, Chap. II]. We note that systems (1.1)-(1.5) are obtained from the original system by a reduction, which is explained in Sect. 3 below and which will be essential for the following analysis. We will only consider the case $\rho(c) \not \equiv$ const., i.e., $\beta \neq 0$. Results for the case of matched densities $(\rho(c) \equiv$ const.) were obtained by Starovoitov [17], Boyer [6], Liu and Shen [12], and the author [1]. Moreover, in [7] Boyer considered a different diffuse interface model for fluids with non-matched densities. He proved existence of strong solutions, locally in time, and existence of global weak solutions if the densities of the fluids are sufficiently close. Finally, A. and Feireisl [3] constructed weak solutions globally in time for a corresponding diffuse interface model for compressible fluids.

We note that the total energy of the system is $E(c, v)=E_{\text {free }}(c)+E_{\text {kin }}(c, v)$, where

$$
E_{\mathrm{free}}(c)=\int_{\Omega} \Phi(c) d x+\int_{\Omega} a(c) \frac{|\nabla c|^{q}}{q} d x, \quad E_{\mathrm{kin}}(c, v)=\int_{\Omega} \rho(c) \frac{|v|^{2}}{2} d x
$$


and that every sufficiently smooth solution of (1.1)-(1.9) satisfies

$$
\begin{aligned}
\frac{d}{d t} E(c(t), v(t)) \\
=-\int_{\Omega}\left(2 v(c)|D v(t)|^{2}+\eta(c)|\operatorname{div} v(t)|^{2}\right) d x \\
\quad-\int_{\Omega} m(c)|\nabla \mu|^{2} d x-\gamma \int_{\partial \Omega}\left|v_{\tau}\right|^{2} d \sigma,
\end{aligned}
$$

cf. (3.12) below. Moreover, note that $a(c)|\nabla c|^{q}=|\nabla A(c)|^{q}$. We will only consider the case $\beta \neq 0$ since $\rho(c) \equiv$ const. if $\beta=0$. Furthermore, we can assume w.l.o.g. that $-\alpha<\beta<0$. Otherwise we replace $c$ with $-c$. Then $\hat{\rho}(s)$ is a strictly increasing function.

Let us comment on the new difficulties that arise for the construction of weak solutions (in comparison with the case of matched densities):

1. Since $\rho=\hat{\rho}(c) \not \equiv$ const. in general, $\operatorname{div} v \not \equiv 0$ too. Therefore it is not sufficient to work in function spaces of divergence free vector fields and we cannot simply apply the Helmholtz projection to (1.1) to get an evolution equation for $v$ independent of $g_{0}$.

2. In the case of non-matched densities $\beta \neq 0$ the (modified) pressure $g_{0}$ enters the equation for the chemical potential (1.4). But $g_{0}$ has low regularity, in particular with respect to time as will be discussed below. Therefore we will not be able to obtain an a priori estimate of $\phi(c)-\Delta c$ in some $L^{r}\left(Q_{T}\right), r \geq 2$, (even not for $r>1$ ). This would be essential to be able to choose $\phi=\Phi^{\prime}$, where $\Phi$ is a singular free energy density as e.g.

$$
\Phi(c)=\frac{\theta}{2}((1+c) \ln (1+c)+(1-c) \ln (1-c))-\frac{\theta_{c}}{2} c^{2},
$$

where $\theta, \theta_{c}>0, a=-1, b=1$. In the case of matched densities $\beta=0$ and with $q=2$, it is possible to show existence of weak solutions such that $c(t, x) \in(-1,1)$ a.e.[1], see also [4]. Since we cannot use these free energy densities, we need some other mechanism to keep the concentration $c \in[-1,1]$ or at least in a suitable neighborhood $c \in[-1-\varepsilon, 1+\varepsilon], \varepsilon>0$. Otherwise, $\rho=\hat{\rho}(c)$ becomes singular or non-positive.

In order to overcome the last difficulty, it will be essential to choose the exponent of the gradient term in the free energy as $q>d$ and to choose the free energy density $\Phi(c)$ "steep enough" outside the interval $[-1,1]$, cf. Lemma 2.3 below. The following proofs will not work for the standard case $q=2$.

For the following analysis it is essential to use a suitable decomposition of $g_{0}$, namely:

$$
g_{0}=g_{1}-\partial_{t} G(v)
$$

where

$$
\begin{gathered}
\Delta G(v)=\operatorname{div} v \quad \text { in } \Omega, \\
\partial_{n} G(v)=0 \quad \text { on } \partial \Omega,
\end{gathered}
$$

and $\int_{\Omega} G(v) d x=0$, which implies that

$$
\nabla G(v)=\left(I-P_{\sigma}\right) v .
$$


Hence (1.1) is equivalent to

$$
\rho \partial_{t} P_{\sigma} v+\rho v \cdot \nabla v-\operatorname{div} S(c, D v)+\rho \nabla g_{1}=\rho \mu_{0} \nabla c \text { in } Q .
$$

Here the part $g_{1}$ has relatively good regularity, e.g., $g_{1} \in L^{2}\left(0, \infty ; L^{p}(\Omega)\right)$ with $1<p<\frac{d}{d-1}$, cf. Lemma 6.1 below. It is the part $\partial_{t} G(v)$, which makes the analysis difficult and which does not allow to use a singular free energy as in (1.11). Finally, let us note that for our estimates of $g_{1}$ it is important to consider Navier boundary conditions for $v$ and not no-slip boundary conditions.

The article is organized as follows: In Sect. 2 we define weak solutions of the system (1.1)-(1.9) and state the main result on existence of weak solutions. In Sect. 3 we state the original form of the system (1.1)-(1.9) as derived in [13], explain the reduction to (1.1)-(1.9), and discuss the conserved quantities. Moreover, in Sect. 4, we introduce the used function spaces and summarize some preliminary results. The existence is proved with the aid of a two-level approximation. Firstly, we add some extra terms depending on a parameter $\delta>0$, which yield an additional a priori bound for $g_{0} \in L^{2}(Q)$. The corresponding system is presented in Sect. 5 and existence of weak solutions to the latter system is proved with the aid of an implicit time discretization, which is the second level of approximation. Then, in Sect. 6, we consider the limit $\delta \rightarrow 0$ and prove our main result on existence of weak solutions to (1.1)-(1.9).

Finally, we note the present results are part of the author's Habilitation thesis [2].

\section{Weak Solutions and the Main Result}

First of all, let us make some basic assumptions:

Assumption 2.1. We assume that $a \in C^{1}(\mathbb{R}), v, \eta, m \in C^{0}(\mathbb{R})$ with $a(s), v(s), m(s) \geq$ $m_{0}>0, \eta(s) \geq 0$ for all $s \in \mathbb{R}$, let $\hat{\rho}(s)=(\alpha+\beta s)^{-1}$ for some $0<-\beta<\alpha$, and let $S(c, D v), \bar{A}(c)$ be defined as in (1.6). Moreover, let $0<\gamma<\infty$ and let $\Omega \subset \mathbb{R}^{d}$, $d=2,3$, be a bounded domain with $C^{2}$-boundary.

In the following, we frequently use the spaces $H_{n}^{1}(\Omega)=\left\{f \in H^{1}(\Omega)^{d}:\left.n \cdot f\right|_{\partial \Omega}=0\right\}$ and $H_{n}^{-1}(\Omega)=\left(H_{n}^{1}(\Omega)\right)^{\prime}$. For more definitions of the used function spaces we refer to Sect. 4. Moreover, we denote $Q_{(s, t)}=\Omega \times(s, t), Q_{t}=Q_{(0, t)}, S_{(s, t)}=\partial \Omega \times(s, t)$, $S_{t}=S_{(0, t)}$ and $A: B=\sum_{j, k=1}^{d} a_{j k} b_{j k}$ for two matrices $A=\left(a_{j k}\right), B=\left(b_{j k}\right) \in \mathbb{R}^{d \times d}$.

Having in mind the decomposition (1.12), we define weak solutions of (1.1)-(1.9) as follows:

Definition 2.2. Let Assumption 2.1 hold, let $v_{0} \in L^{2}(\Omega)^{d}, c_{0} \in W_{q}^{1}(\Omega), q>d$, let $\Phi: \mathbb{R} \rightarrow[0, \infty)$ be twice continuously differentiable and set $\phi=\Phi^{\prime}$. Then $\left(v, g_{1}, c, \mu_{0}, \bar{p}\right)$ with

$$
\begin{aligned}
v & \in B C_{w}\left([0, \infty) ; L^{2}(\Omega)^{d}\right) \cap L^{2}\left(0, \infty ; H_{n}^{1}(\Omega)\right), \\
g_{1} & \in L^{2}\left(0, \infty ; L_{(0)}^{1}(\Omega)\right), \quad c \in B C_{w}\left([0, \infty) ; W_{q}^{1}(\Omega)\right), \\
\mu_{0} & \in L^{2}\left(0, \infty ; H^{1}(\Omega)\right), \quad \bar{p} \in L_{l o c}^{1}(0, \infty),
\end{aligned}
$$

and such that $0<\rho=\hat{\rho}(c) \in L^{\infty}(Q)$ is called a weak solution of (1.1)-(1.9) if the following conditions are satisfied: 
1. For every $\varphi \in C_{0}^{\infty}\left(0, \infty ; H_{n}^{1}(\Omega) \cap L^{\infty}(\Omega)^{d}\right)$,

$$
\begin{aligned}
& -\left(P_{\sigma} v, \partial_{t} \varphi\right)_{Q}+(v \cdot \nabla v, \varphi)_{Q}+\left(\rho^{-1} S(c, D v), D \varphi\right)_{Q} \\
& \quad+\gamma\left(\rho^{-1} v_{\tau}, \varphi_{\tau}\right)_{S}=\left(g_{1}, \operatorname{div} \varphi\right)_{Q}+\left(\mu_{0} \nabla c, \varphi\right)_{Q}-\left(\nabla \rho^{-1} \cdot S(c, D v), \varphi\right)_{Q} .
\end{aligned}
$$

2. For every $\psi \in C_{0}^{\infty}\left(0, \infty ; C^{1}(\bar{\Omega})\right)$,

$$
\begin{gathered}
\left(\rho, \partial_{t} \psi\right)_{Q}+(\rho v, \nabla \psi)_{Q}=0, \\
\left(\rho c, \partial_{t} \psi\right)_{Q}+(\rho c v, \nabla \psi)_{Q}=(m(c) \nabla \mu, \nabla \psi)_{Q},
\end{gathered}
$$

and

$$
\begin{aligned}
& \left(a(c)^{-\frac{1}{q}}\left(\rho \mu_{0}+\rho^{2} \bar{p}-\phi(c)\right), \psi\right)_{Q}=\beta\left(a(c)^{-\frac{1}{q}} \rho^{2} g_{1}, \psi\right)_{Q} \\
& -\beta\left(G(v), \partial_{t}\left(a(c)^{-\frac{1}{q}} \rho^{2} \psi\right)\right)_{Q}+\left(|\nabla A(c)|^{q-2} \nabla A(c), \nabla \psi\right)_{Q} .
\end{aligned}
$$

3. $\left.(v, c)\right|_{t=0}=\left(v_{0}, c_{0}\right)$.

4. $(v, c, \mu)$ satisfy the energy inequality

$$
\begin{aligned}
& E(c(t), v(t))+ \\
& \int_{Q_{(s, t)}}\left(S(c, D v): D v+m(c)|\nabla \mu|^{2}\right) d(x, \tau)+\gamma\left\|v_{\tau}\right\|_{L^{2}\left(S_{(s, t)}\right)}^{2} \leq E(c(s), v(s))
\end{aligned}
$$

for all $t \in[s, \infty)$ and almost all $0 \leq s<\infty$ including $s=0$.

We note that the boundary conditions (1.8) and the second condition in (1.7) are part of the weak formulations (2.1)-(2.3) because of

$$
\begin{aligned}
-(\operatorname{div} S(c, D v), \varphi)_{\Omega} & =(S(c, D v), \nabla \varphi)_{\Omega}-(n \cdot S(c, D v), \varphi)_{\partial \Omega} \\
& =(S(c, D v), \nabla \varphi)_{\Omega}+\gamma(v(c) v, \varphi)_{\partial \Omega},
\end{aligned}
$$

for all $v \in H^{2}(\Omega)$ satisfying (1.7) and all $\varphi \in H_{n}^{1}(\Omega)$.

The following lemma shows that, given $\Phi \in C^{2}([-1,1])$ and $R, \varepsilon>0$, we can "trap" all $c(x)$ with $E_{\text {free }}(c) \leq R$ in an arbitrary small neighborhood $(-1-\varepsilon, 1+\varepsilon)$ by extending $\Phi$ "suitably steep" outside of $[-1,1]$. This is essential for everything that follows.

Lemma 2.3. Let $R, \varepsilon>0, q>d$, and let $\Phi \in C^{2}([-1,1])$ with $\Phi(c)>0, c \in[-1,1]$, be given. Then there is an extension $\Phi \in C^{2}(\mathbb{R}), \Phi(c) \geq 0, \Phi^{\prime \prime}(c) \geq-M>-\infty$ such that for all $c \in W_{q}^{1}(\Omega)$,

$$
\int_{\Omega} \frac{a(c)|\nabla c|^{q}}{q} d x+\int_{\Omega} \Phi(c) d x \leq R \Rightarrow c(x) \in(-1-\varepsilon, 1+\varepsilon) .
$$

Proof. Since $W_{q}^{1}(\Omega) \hookrightarrow C^{\alpha}(\bar{\Omega}), \alpha=1-\frac{d}{q}$, there is a constant $C_{1}$ such that

$$
\left|c(x)-c\left(x_{0}\right)\right| \leq C_{1}\|\nabla c\|_{L^{q}(\Omega)}\left|x-x_{0}\right|^{\alpha}
$$


for all $x, x_{0} \in \bar{\Omega}$ and $c \in W_{q}^{1}(\Omega)$ with $\int_{\Omega} c d x=0$. Since we can change $c(x)$ by a constant in the estimate above, it also holds for all $c \in W_{q}^{1}(\Omega)$. Moreover, if $E_{\text {free }}(c) \leq R$, then $\|\nabla c\|_{L^{q}} \leq R^{\prime}$ for some $R^{\prime}>0$. Hence $\left|c(x)-c\left(x_{0}\right)\right| \leq C_{1} R^{\prime}\left|x-x_{0}\right|^{\alpha} \leq \frac{\varepsilon}{2} \quad$ for all $x \in B_{r}\left(x_{0}\right) \cap \bar{\Omega}, r:=\left(\frac{\varepsilon}{2 C_{1} R^{\prime}}\right)^{\frac{1}{\alpha}}$.

Furthermore, let $\kappa=\inf _{x_{0} \in \bar{\Omega}}\left|B_{r}\left(x_{0}\right) \cap \Omega\right|>0$. Hence for every $x_{0} \in \bar{\Omega}$ we have $|c(x)| \geq\left|c\left(x_{0}\right)\right|-\frac{\varepsilon}{2}$ on a set of at least measure $\kappa$.

Now we choose the extension of $\Phi \in C^{2}([-1,1]), \Phi(s)>0$, to $\Phi: \mathbb{R} \rightarrow[0, \infty)$ such that $\Phi(s) \geq M:=\kappa^{-1}(R+1)$ and $\Phi^{\prime \prime}(s) \geq 0$ for all $|s| \geq 1+\frac{\varepsilon}{2}$. In order to prove (2.7), we assume that for $c \in W_{q}^{1}(\Omega)$ there is some $x_{0} \in \bar{\Omega}$ such that $\left|c\left(x_{0}\right)\right| \geq 1+\varepsilon$. By the observations before, we conclude that $|c(x)| \geq\left|c\left(x_{0}\right)\right|-\frac{\varepsilon}{2} \geq 1+\frac{\varepsilon}{2}$ on a set $S=B_{r}\left(x_{0}\right) \cap \Omega$ of measure at least $\kappa>0$. Hence

$$
E_{\text {free }}(c) \geq \int_{S} \Phi(c) d x \geq \kappa M=R+1,
$$

which proves the implication (2.7).

Now we are able to state our main result on existence of weak solutions.

Theorem 2.4. Let $q>d, \varepsilon, R>0$. Moreover, let $\Phi \in C^{2}(\mathbb{R}), \Phi(c) \geq 0, \Phi^{\prime \prime}(c) \geq$ $-M$, be given such that (2.7) holds. Then for every $v_{0} \in L^{2}(\Omega)^{d}, c_{0} \in W_{q}^{1}(\Omega)$ with $E\left(c_{0}, v_{0}\right) \leq R$ there exists a weak solution $\left(v, g_{1}, c, \mu_{0}, \bar{p}\right)$ of $(1.1)-(1.9)$ in the sense of Definition 2.2 and with the property that

$$
\begin{array}{cl}
c(t, x) \in[-1-\varepsilon, 1+\varepsilon] & \text { for all }(x, t) \in \bar{Q}, \\
g_{1} \in L^{2}\left(0, \infty ; L^{p}(\Omega)\right), & \bar{p} \in L_{\mathrm{uloc}}^{2}([0, \infty))
\end{array}
$$

for every $1<p<\frac{d}{d-1}$.

\section{Reformulation of the System and Conserved Quantities}

Our starting point is the system

$$
\begin{gathered}
\rho \partial_{t} v+\rho v \cdot \nabla v-\operatorname{div} S(c, D v)+\nabla p=-\operatorname{div}\left(|\nabla A(c)|^{q-2} \nabla A(c) \otimes \nabla A(c)\right), \\
\partial_{t} \rho+\operatorname{div}(\rho v)=0, \\
\rho \partial_{t} c+\rho v \cdot \nabla c=\operatorname{div}(m(c) \nabla \mu), \\
\rho \mu=-\rho^{-1} \frac{\partial \rho}{\partial c}\left(p+\Phi(c)+\frac{|\nabla A(c)|^{q}}{q}\right)+\phi(c)-a(c)^{\frac{1}{q}} \Delta_{q} A(c),
\end{gathered}
$$

which is a variant of the model proposed in [13] for an interfacial energy of the form

$$
E_{\text {free }}(c)=\int_{\Omega} \Phi(c) d x+\int_{\Omega} a(c) \frac{|\nabla c|^{q}}{q} d x
$$


where the choice $q=2, a(c)=\rho(c)$ was proposed in the latter article. A derivation of the latter system can also be found in [2, Chap. II]. We close the system by adding initial and boundary conditions (1.7)-(1.9). We note that the term $|\nabla A(c)|^{q-2} \nabla A(c) \otimes \nabla A(c)$ comes from an extra contribution to the stress tensor, which models capillary forces in an interfacial region.

In order to derive (1.1)-(1.5), we define

$$
g=\frac{\Phi(c)}{\rho}+\frac{|\nabla A(c)|^{q}}{\rho q}+\frac{p}{\rho}-\bar{\mu} c,
$$

where $\mu=\mu_{0}+\bar{\mu}$ and $\bar{\mu}=\frac{1}{|\Omega|} \int_{\Omega} \mu d x$. Then

$\rho \nabla g=\phi(c) \nabla c+\nabla \frac{|\nabla A(c)|^{q}}{q}+\nabla p-\rho^{-1} \frac{\partial \rho}{\partial c}\left(p+\Phi(c)+\frac{|\nabla A(c)|^{q}}{q}\right) \nabla c-\rho \bar{\mu} \nabla c$,

and therefore

$$
\begin{aligned}
\rho \nabla g-\rho \mu_{0} \nabla c & =\nabla p+a(c)^{\frac{1}{q}} \Delta_{q} A(c) \nabla c+\nabla \frac{|\nabla A(c)|^{q}}{q} \\
& =\nabla p+\operatorname{div}\left(|\nabla A(c)|^{q-2} \nabla A(c) \otimes \nabla A(c)\right) .
\end{aligned}
$$

Hence we see that (3.1)-(3.4) is equivalent to the system

$$
\begin{gathered}
\rho \partial_{t} v+\rho v \cdot \nabla v-\operatorname{div} S(c, D v)+\rho \nabla g=\rho \mu_{0} \nabla c \text { in } Q, \\
\partial_{t} \rho+\operatorname{div}(\rho v)=0 \quad \text { in } Q, \\
\rho \partial_{t} c+\rho v \cdot \nabla c=\operatorname{div}(m(c) \nabla \mu) \quad \text { in } Q,
\end{gathered}
$$

and

$$
\rho \mu_{0}+\left(\rho+\frac{\partial \rho}{\partial c} c\right) \bar{\mu}=-\frac{\partial \rho}{\partial c} g-a^{\frac{1}{q}}(c) \Delta_{q} A(c)+\phi(c) .
$$

For the following mathematical analysis it will be essential to consider the case of a so-called simple mixture. This is expressed in the relation

$$
\frac{1}{\rho}=\frac{c_{1}}{\bar{\rho}_{1}}+\frac{c_{2}}{\bar{\rho}_{2}},
$$

which means that the volume of the mixed fluids is the same as the sum of the volume filled by the separated fluids. Here $c_{j}$ is the concentration and $\bar{\rho}_{j}$ is the mass density of the fluid $j=1,2$. Therefore $\hat{\rho}$ can be written in the form

$$
\hat{\rho}(c)=\frac{1}{\alpha+\beta c} \quad \text { where } \beta=\frac{1}{2 \bar{\rho}_{1}}-\frac{1}{2 \bar{\rho}_{2}}, \alpha=\frac{1}{2 \bar{\rho}_{2}}+\frac{1}{2 \bar{\rho}_{1}}
$$

with $\alpha>0$ and $|\beta|<\alpha$. One easily calculates that

$$
\frac{\partial \rho}{\partial c}=-\beta \rho^{2}, \quad \rho+\frac{\partial \rho}{\partial c} c=\alpha \rho^{2} .
$$

Therefore (3.8) reduces to

$$
\rho \mu_{0}+\rho^{2}(\alpha \bar{\mu}+\beta \bar{g})=\beta \rho^{2} g_{0}-a^{\frac{1}{q}}(c) \Delta_{q} A(c)+\phi(c),
$$


where $g=g_{0}+\bar{g}$ and $\bar{g}=\frac{1}{|\Omega|} \int_{\Omega} g(x) d x$. Hence one sees that $\bar{\mu}, \bar{g}$ are not uniquely determined by the system. But $\bar{p}=\alpha \bar{\mu}+\beta \bar{g}$ is determined uniquely by (3.11) if $\mu_{0}, g_{0}$ and $c$ are known. A closely related identity is

$$
\partial_{t} \rho=-\beta \rho^{2} \partial_{t} c=-\frac{\beta}{\alpha}\left(\rho+\frac{\partial \rho}{\partial c} c\right) \partial_{t} c=-\frac{\beta}{\alpha} \partial_{t}(\rho c) .
$$

In particular, this implies

$$
\frac{d}{d t} \int_{\Omega} \rho c d x=0 \Rightarrow \frac{d}{d t} \int_{\Omega} \rho d x=0 .
$$

Summing up, we have derived (1.1)-(1.5) from the original system (3.1)-(3.4) assuming the case of a simple mixture.

Now we discuss the conserved quantities and the energy estimate for solutions of the system. Because of (3.2), (3.3) is equivalent to

$$
\partial_{t}(\rho c)+\operatorname{div}(\rho c v)=\operatorname{div}(m(c) \nabla \mu) .
$$

Therefore the total mass difference $\rho c$ and the total mass are conserved:

$$
\begin{aligned}
\int_{\Omega} \rho(x, t) c(x, t) d x & =\int_{\Omega} \rho_{0}(x) c_{0}(x) d x \quad \text { for all } t \in(0, \infty), \\
\int_{\Omega} \rho(x, t) d x & =\int_{\Omega} \rho_{0}(x) d x \quad \text { for all } t \in(0, \infty) .
\end{aligned}
$$

Moreover, multiplying (3.5) with $v$, (3.7) with $\mu$, and (3.8) with $\partial_{t} c$, integrating with respect to $\Omega$, and using the boundary conditions and (3.6), one sees that every sufficiently smooth solution of (1.1)-(1.9) satisfies

$$
\begin{aligned}
\frac{d}{d t} E(c(t), v(t))= & -\int_{\Omega}\left(2 v(c(t))|D v(t)|^{2}+\eta(c(t))|\operatorname{div} v(t)|^{2}\right) d x \\
& -\int_{\Omega} m(c)|\nabla \mu|^{2} d x-\int_{\partial \Omega} \gamma|v|^{2} d \sigma,
\end{aligned}
$$

where $E(c, v)=E_{\text {free }}(c)+E_{\text {kin }}(c, v)$ and $E_{\text {free }}(c), E_{\text {kin }}(c, v)$ are defined as in (1.10). Integrating (3.12) yields (2.5).

Finally, we note that (3.6) and (3.10) imply

$$
-\beta \rho^{2}\left(\partial_{t} c+v \cdot \nabla c\right)=-\rho \operatorname{div} v
$$

Combining this with (3.7), we obtain the simple identity

$$
\operatorname{div} v=\beta \operatorname{div}\left(m(c) \nabla \mu_{0}\right) .
$$




\section{Preliminaries}

Notation. Let us fix some notation. If $X$ is a Banach space and $X^{\prime}$ is its dual, then

$$
\langle f, g\rangle \equiv\langle f, g\rangle_{X^{\prime}, X}=f(g), \quad f \in X^{\prime}, g \in X,
$$

denotes the duality product. The inner product on a Hilbert space $H$ is denoted by $(., .)_{H}$. Moreover, we use the abbreviation (., . $)_{M}=(.,)_{L^{2}(M)}$.

Function spaces. If $M \subseteq \mathbb{R}^{d}$ is measurable, $L^{q}(M), 1 \leq q \leq \infty$ denotes the usual Lebesgue-space and $\|.\|_{q}$ its norm. Moreover, $L^{q}(M ; X)$ denotes its vector-valued variant of strongly measurable $q$-integrable functions/essentially bounded functions, where $X$ is a Banach space. If $M=(a, b)$, we write for simplicity $L^{q}(a, b ; X)$ and $L^{q}(a, b)$. Furthermore, $f \in L_{\mathrm{loc}}^{q}([0, \infty) ; X)$ if and only if $f \in L^{q}(0, T ; X)$ for every $T>0$. Moreover, $L_{\mathrm{uloc}}^{q}([0, \infty) ; X)$ denotes the uniformly local variant of $L^{q}(0, \infty ; X)$ consisting of all measurable $f:[0, \infty) \rightarrow X$ such that

$$
\|f\|_{L_{\text {uloc }}^{q}([0, \infty) ; X)}=\sup _{t \geq 0}\|f\|_{L^{q}(t, t+1 ; X)}<\infty .
$$

Recall that, if $X$ is a Banach space with the Radon-Nikodym property, then

$$
L^{q}(M ; X)^{\prime}=L^{q^{\prime}}\left(M ; X^{\prime}\right) \quad \text { for every } 1 \leq q<\infty
$$

by means of the duality product $\langle f, g\rangle=\int_{M}\langle f(x), g(x)\rangle_{X^{\prime}, X} d x$ for $f \in L^{q^{\prime}}\left(M ; X^{\prime}\right)$, $g \in L^{q}(M ; X)$. If $X$ is reflexive or $X^{\prime}$ is separable, then $X$ has the Radon-Nikodym property, cf. Diestel and Uhl [9].

Moreover, recall the lemma of Aubin-Lions: If $X_{0} \hookrightarrow \hookrightarrow X_{1} \hookrightarrow X_{2}$ are Banach spaces, $1<p<\infty, 1 \leq q<\infty$, and $I \subset \mathbb{R}$ is a bounded interval, then

$$
\left\{v \in L^{p}\left(I ; X_{0}\right): \frac{d v}{d t} \in L^{q}\left(I ; X_{2}\right)\right\} \hookrightarrow \hookrightarrow L^{p}\left(I ; X_{1}\right) .
$$

See J.-L. Lions [11] for the case $q>1$ and Simon [16] or Roubíček [14] for $q=1$.

Let $\Omega \subset \mathbb{R}^{d}$ be a domain. Then $W_{q}^{m}(\Omega), m \in \mathbb{N}_{0}, 1 \leq q \leq \infty$, denotes the usual $L^{q}$-Sobolev space, $W_{q, 0}^{m}(\Omega)$ the closure of $C_{0}^{\infty}(\Omega)$ in $W_{q}^{m}(\Omega), W_{q}^{-m}(\Omega)=\left(W_{q^{\prime}, 0}^{m}(\Omega)\right)^{\prime}$, and $W_{q, 0}^{-m}(\Omega)=\left(W_{q^{\prime}}^{m}(\Omega)\right)^{\prime}$. The $L^{2}$-Bessel potential spaces are denoted by $H^{s}(\Omega)$, $s \in \mathbb{R}$, which are defined by restriction of distributions in $H^{s}\left(\mathbb{R}^{d}\right)$ to $\Omega$, cf. Triebel [19, Sect. 4.2.1]. We note that, if $\Omega \subset \mathbb{R}^{d}$ is a bounded domain with $C^{0,1}$-boundary, then there is an extension operator $E_{\Omega}$ which is a bounded linear operator $E_{\Omega}: W_{p}^{m}(\Omega) \rightarrow$ $W_{p}^{m}\left(\mathbb{R}^{d}\right), 1 \leq p \leq \infty$ for all $m \in \mathbb{N}$ and $\left.E_{\Omega} f\right|_{\Omega}=f$ for all $f \in W_{p}^{m}(\Omega)$, cf. Stein [18, Chap. VI, Sect. 3.2]. This extension operator extends to $E_{\Omega}: H^{s}(\Omega) \rightarrow H^{s}\left(\mathbb{R}^{n}\right)$, which shows that $H^{s}(\Omega)$ is a retract of $H^{s}(\Omega)$. Therefore all results on interpolation spaces of $H^{s}\left(\mathbb{R}^{n}\right)$ carry over to $H^{s}(\Omega)$.

Furthermore, we note that

$$
\|f g\|_{W_{p}^{1}} \leq C_{p, q}\|f\|_{W_{q}^{1}}\|g\|_{W_{p}^{1}} \quad \text { for all } 1<p \leq q \leq \infty, q>d,
$$

which is easily proved using well-known Sobolev embeddings.

Let $I=[0, T]$ with $0<T<\infty$ or let $I=[0, \infty)$ and let $X$ be a Banach space. Then $B C(I ; X)$ is the Banach space of all bounded and continuous $f: I \rightarrow X$ equipped with the supremum norm and $B U C(I ; X)$ is the subspace of all bounded and uniformly 
continuous functions. Moreover, we define $B C_{w}(I ; X)$ as the topological vector space of all bounded and weakly continuous functions $f: I \rightarrow X$. By $C_{0}^{\infty}(0, T ; X)$ we denote the vector space of all smooth functions $f:(0, T) \rightarrow X$ with supp $f \subset \subset(0, T)$. Finally, $f \in W_{p}^{1}(0, T ; X), 1 \leq p<\infty$ if and only if $f, \frac{d f}{d t} \in L^{p}(0, T ; X)$, where $\frac{d f}{d t}$ denotes the vector-valued distributional derivative of $f$. Furthermore, $W_{p \text {, uloc }}^{1}([0, \infty) ; X)$ is defined by replacing $L^{p}(0, T ; X)$ by $L_{\text {uloc }}^{p}([0, \infty) ; X)$ and we set $H^{1}(0, T ; X)=W_{2}^{1}(0, T ; X)$.

Finally, we note:

Lemma 4.1. Let $X, Y$ be two Banach spaces such that $Y \hookrightarrow X$ and $X^{\prime} \hookrightarrow Y^{\prime}$ densely and let $0<T<\infty$. Then $L^{\infty}(0, T ; Y) \cap B U C([0, T] ; X) \hookrightarrow B C_{w}([0, T] ; Y)$.

Proof. If $f \in L^{\infty}(0, T ; Y) \cap B U C([0, T] ; X)$, then $\langle f(t), \varphi\rangle_{X, X^{\prime}} \in B U C([0, T])$ for all $\varphi \in X^{\prime}$. Now let $\varphi \in Y^{\prime}$. Since $X^{\prime} \hookrightarrow Y^{\prime}$ densely, we can find a sequence $\varphi_{k} \in X^{\prime}$ such that $\varphi_{k} \rightarrow_{k \rightarrow \infty} \varphi$ in $Y^{\prime}$. Because of $f \in L^{\infty}(0, T ; Y)$, this implies that $\left\langle f(t), \varphi_{k}\right\rangle_{Y, Y^{\prime}} \rightarrow$ $\langle f(t), \varphi\rangle_{Y, Y^{\prime}}$ uniformly in $[0, T]$. Hence $\langle f(t), \varphi\rangle_{Y, Y^{\prime}} \in B U C([0, T])$.

Neumann-Laplace equation. Given $f \in L^{1}(\Omega)$, we denote by $m(f)=\frac{1}{|\Omega|} \int_{\Omega} f(x) d x$ its mean value. Moreover, for $m \in \mathbb{R}$ we set

$$
L_{(m)}^{q}(\Omega):=\left\{f \in L^{q}(\Omega): m(f)=m\right\}, \quad 1 \leq q \leq \infty .
$$

Then

$$
P_{0} f:=f-m(f)=f-\frac{1}{|\Omega|} \int_{\Omega} f(x) d x
$$

is the orthogonal projection onto $L_{(0)}^{2}(\Omega)$. Furthermore, we define

$$
H_{(0)}^{1} \equiv H_{(0)}^{1}(\Omega)=H^{1}(\Omega) \cap L_{(0)}^{2}(\Omega), \quad(c, d)_{H_{(0)}^{1}(\Omega)}:=(\nabla c, \nabla d)_{L^{2}(\Omega)} .
$$

Then $H_{(0)}^{1}(\Omega)$ is a Hilbert space due to Poincaré's inequality. Moreover, let $H_{(0)}^{-1} \equiv H_{(0)}^{-1}(\Omega)=H_{(0)}^{1}(\Omega)^{\prime}$. Furthermore, we define $\operatorname{div}_{n}: L^{2}(\Omega)^{d} \rightarrow H_{(0)}^{-1}(\Omega)$ by

$$
\left\langle\operatorname{div}_{n} u, \varphi\right\rangle_{H_{(0)}^{-1}, H_{(0)}^{1}}=-(u, \nabla \varphi)_{L^{2}(\Omega)} \quad \text { for all } \varphi \in H_{(0)}^{1}(\Omega) .
$$

Note that this operator should not be confused with the distributional divergence extended to an operator div: $L^{2}(\Omega)^{d} \rightarrow H^{-1}(\Omega)=\left(H_{0}^{1}(\Omega)\right)^{\prime}$, since the latter is defined only for $C_{0}^{\infty}(\Omega)$ - resp. $H_{0}^{1}(\Omega)$-vector fields. The operator $\operatorname{div}_{n}$ acts on vector fields, which do not vanish on the boundary necessarily, and involves boundary conditions in a weak sense.

Let $m \in L^{\infty}(\Omega)$ such that, $m(x) \geq m_{0}>0$ a.e. Then $\operatorname{div}_{n}(m(x) \nabla \cdot): H_{(0)}^{1}(\Omega) \rightarrow$ $H_{(0)}^{-1}(\Omega)$, defined by

$$
-\left\langle\operatorname{div}_{n}(m(x) \nabla u), \varphi\right\rangle_{H_{(0)}^{1}, H_{(0)}^{-1}}=(m(x) \nabla u, \nabla \varphi) \quad \text { for all } \varphi \in H_{(0)}^{1}(\Omega)
$$

is an isomorphism because of the lemma of Lax-Milgram. In particular, this is true for the weak Neumann-Laplace operator $\Delta_{N}:=\operatorname{div}_{n} \nabla$ and $\Delta_{N} u=f$ for $u \in H_{(0)}^{1}(\Omega)$, $f \in H_{(0)}^{-1}(\Omega)$ implies

$$
\|u\|_{H_{(0)}^{1}(\Omega)} \leq\|f\|_{H_{(0)}^{-1}(\Omega)}
$$


Finally, we note that, if $u \in H_{(0)}^{1}(\Omega)$ solves $\Delta_{N} u=f$ for some $f \in L_{(0)}^{q}(\Omega), 1<q<$ $\infty$, and $\Omega$ is a bounded domain with $C^{2}$-boundary, then it follows from standard elliptic theory that $u \in W_{q}^{2}(\Omega)$ and $\Delta u=f$ a.e. in $\Omega$ and $\left.\partial_{n} u\right|_{\partial \Omega}=0$ in the sense of traces. Moreover,

$$
\|u\|_{W_{q}^{2}(\Omega)} \leq C_{q}\|f\|_{L^{q}(\Omega)} \quad \text { for all } f \in L_{(0)}^{q}(\Omega)
$$

with a constant $C_{q}$ depending only on $1<q<\infty, d$, and $\Omega$.

For the following we denote

$$
W_{p, N}^{2}(\Omega)=\left\{u \in W_{p}^{2}(\Omega):\left.\partial_{n} u\right|_{\partial \Omega}=0\right\},
$$

where $1<p<\infty$.

By a simple duality argument one can define so-called very weak solutions of the Neumann-Laplace operator, which is the content of the next lemma.

Lemma 4.2. Let $1<p<\infty$. Then for every $f \in\left(W_{p^{\prime}, N}^{2}(\Omega)\right)^{\prime}$ there is a unique $u \in L^{p}(\Omega)$ such that

$$
(u, \Delta \varphi)_{\Omega}=\langle f, \varphi\rangle_{\left(W_{p^{\prime}, N}^{2}\right)^{\prime}, W_{p^{\prime}, N}^{2}}
$$

for all $\varphi \in W_{p^{\prime}, N}^{2}(\Omega)$. Moreover, there is some $C_{p}$ such that

$$
\|u\|_{L^{p}(\Omega)} \leq C_{p}\|f\|_{\left(W_{p^{\prime}, N}^{2}(\Omega)\right)^{\prime}}
$$

for all $u \in L^{p}(\Omega)$ and $f \in\left(W_{p^{\prime}, N}^{2}(\Omega)\right)^{\prime}$, solving (4.6).

Proof. Since $\Delta_{N}: W_{p^{\prime}, N}^{2}(\Omega) \rightarrow L^{p^{\prime}}(\Omega)$ is a bijection, the adjoint $\Delta_{N}^{\prime}: L^{p}(\Omega) \rightarrow$ $\left(W_{p^{\prime}, N}^{2}(\Omega)\right)^{\prime}$ is a bijection too. Hence for every $f \in\left(W_{p^{\prime}, N}^{2}(\Omega)\right)^{\prime}$ there is a unique $u \in L^{p}(\Omega)$ such that $\Delta_{N}^{\prime} u=f$. Moreover, (4.7) holds since $\left(\Delta_{N}^{\prime}\right)^{-1}$ is continuous. Then

$$
(u, \Delta \varphi)_{\Omega}=\left(u, \Delta_{N} \varphi\right)_{\Omega}=\left\langle\Delta_{N} u, \varphi\right\rangle_{\left(W_{p^{\prime}, N}^{2}(\Omega)\right)^{\prime}, W_{p^{\prime}, N}^{2}(\Omega)}=\langle f, \varphi\rangle_{\left(W_{p^{\prime}, N}^{2}(\Omega)\right)^{\prime}, W_{p^{\prime}, N}^{2}(\Omega)}
$$

for all $\varphi \in W_{p^{\prime}, N}^{2}(\Omega)$ by the definition of the adjoint.

A result related to energy inequalities. The following lemma will be useful for passing to the limit in energy inequalities.

Lemma 4.3. Let $E:[0, T) \rightarrow[0, \infty), 0<T \leq \infty$, be a lower semi-continuous function and let $D:(0, T) \rightarrow[0, \infty)$ be an integrable function. Then

$$
E(0) \varphi(0)+\int_{0}^{T} E(t) \varphi^{\prime}(t) d t \geq \int_{0}^{T} D(t) \varphi(t) d t
$$

holds for all $\varphi \in W_{1}^{1}(0, T)$ with $\varphi(T)=0$ if and only if

$$
E(t)+\int_{s}^{t} D(\tau) d \tau \leq E(s)
$$

holds for all $s \leq t<T$ and almost all $0 \leq s<T$ including $s=0$. 
Proof. First assume that (4.8) holds. If $s=0$ and $0 \leq t<T$, then we choose

$$
\varphi_{\varepsilon, t}(\tau)= \begin{cases}1 & \text { if } 0 \leq \tau<t \\ 1-\frac{1}{\varepsilon}(\tau-t) & \text { if } t \leq \tau \leq t+\varepsilon \\ 0 & \text { otherwise }\end{cases}
$$

in (4.8). Then

$$
\int_{0}^{T} E(\tau) \varphi_{\varepsilon}^{\prime}(\tau) d \tau=\frac{1}{\varepsilon} \int_{t}^{t+\varepsilon} E(\tau) d \tau \rightarrow_{\varepsilon \rightarrow 0} E(t)
$$

for every Lebesgue point $t$ of $E$ (hence almost everywhere). This implies that (4.9) holds for $s=0$ and all Lebesgue points $0 \leq t<\infty$. Since $E$ is lower semi-continuous, we have

$$
E(t) \leq \liminf _{t^{\prime} \rightarrow t} E\left(t^{\prime}\right)
$$

Therefore, choosing a sequence of Lebesgue points $t_{j}^{\prime} \rightarrow t$, we conclude that (4.9) holds for all $0 \leq t<\infty$. In order to prove (4.9) one chooses $\varphi=\varphi_{\varepsilon, t}-\varphi_{\varepsilon, s}$ in (4.8) and proceeds as before.

Conversely, assume that (4.9) holds for all $s \in M$ with $|[0, T] \backslash M|=0$. Then (4.8) holds for every $\varphi \in W_{1}^{1}(0, T)$ such that $\varphi(T)=0$ and $\varphi^{\prime}(\tau)$ is piecewise constant on some intervals $\left[t_{k}, t_{k+1}\right], k=0, \ldots, N, 0=t_{0}<t_{1}<\cdots<t_{N+1}=T$, where $t_{k} \in M$, $k=0, \ldots, N+1$. Since every $\varphi^{\prime} \in L^{1}(0, T)$ can be approximated by piecewise constant functions of the latter form, we conclude that (4.8) holds.

Monotone Operators. Let $X$ be a real-valued Banach space. Recall that $A: X \rightarrow X^{\prime}$ is a monotone operator if and only if

$$
\langle A(x)-A(y), x-y\rangle_{X^{\prime}, X} \geq 0 \quad \text { for all } x, y \in X .
$$

Moreover, a monotone operator $A$ is maximal monotone if for every $w, x \in X$ such that

$$
\langle w-A(y), x-y\rangle_{X^{\prime}, X} \geq 0 \quad \text { for all } y \in X
$$

$w=A(x)$ holds.

An important consequence of (4.10) is the following lemma.

Lemma 4.4. Let $A: X \rightarrow X^{\prime}$ be a maximal monotone operator. Assume that $x_{n}, x \in X$, $y \in X^{\prime}, n \in \mathbb{N}$, satisfy $x_{n} \rightarrow_{n \rightarrow \infty} x, A\left(x_{n}\right) \rightarrow_{n \rightarrow \infty} y$, and $\lim \sup _{n \rightarrow \infty}\left\langle A\left(x_{n}\right), x_{n}\right\rangle \leq$ $\langle y, x\rangle$. Then $y=A(x)$.

The result follows from the fact that a maximal monotone operator is of "type M", cf. [15, Sect. II.2].

We define the $q$-Laplacian $\Delta_{q}: W_{q}^{1}(\Omega) \rightarrow W_{q, 0}^{-1}(\Omega)=\left(W_{q^{\prime}}^{1}(\Omega)\right)^{\prime}, 1<q<\infty$, by

$$
-\left\langle\Delta_{q} u, \varphi\right\rangle_{W_{q^{\prime}, 0}^{-1}, W_{q}^{1}}=\int_{\Omega}|\nabla u|^{q-2} \nabla u \cdot \nabla \varphi d x \quad \text { for all } \varphi \in W_{q}^{1}(\Omega) .
$$

It is easy to check that $-\Delta_{q} u \in \partial \varphi(u)$, where $\varphi: W_{q, 0}^{1}(\Omega) \rightarrow \mathbb{R}$ is defined by

$$
\varphi(u)=\frac{1}{q}\|\nabla u\|_{L^{q}(\Omega)}^{q}
$$


and $w \in \partial \varphi(u) \subseteq W_{q, 0}^{-1}(\Omega)$ if and only if

$$
\langle w, v-u\rangle_{W_{q, 0}^{-1}, W_{q}^{1}} \leq \varphi(v)-\varphi(u) \quad \text { for all } v \in W_{q}^{1}(\Omega) \text {. }
$$

(Consider e.g. the function $f(t)=\varphi((1-t) u+t v), t \in \mathbb{R}$, and use that $f^{\prime}(t)$ is non-decreasing since $f(t)$ is convex.) In particular, this implies that $-\Delta_{q}: W_{q}^{1}(\Omega) \rightarrow$ $W_{q, 0}^{-1}(\Omega)$ is a monotone operator. Moreover, $-\Delta_{q}$ is even strictly monotone, i.e.,

$$
\left\langle\Delta_{q} u-\Delta_{q} v, u-v\right\rangle_{W_{q^{\prime}, 0}^{-1}, W_{q}^{1}}=0 \text { if and only if } u=v
$$

which follows from the fact that $x \mapsto \frac{1}{q}|x|^{q}$ is strictly convex. Furthermore, we note that

$$
\lambda(u, u)_{L^{2}}+\left\langle-\Delta_{q} u, u\right\rangle_{W_{q^{\prime}, 0}^{-1}, W_{q}^{1}}=|\lambda|\|u\|_{L^{2}(\Omega)}^{2}+\|\nabla u\|_{L^{q}(\Omega)}^{q} .
$$

Hence $\lambda-\Delta_{q}$ is coercive on $W_{q}^{1}(\Omega)$ for every $\lambda>0$ if $q \geq 2$, i.e.,

$$
\frac{\lambda(u, u)_{L^{2}}+\left\langle-\Delta_{q} u, u\right\rangle_{W_{q^{\prime}, 0}^{-1}, W_{q}^{1}}}{\|u\|_{W_{q}^{1}(\Omega)}} \rightarrow \infty \quad \text { as }\|u\|_{W_{q}^{1}(\Omega)} \rightarrow \infty .
$$

Therefore we obtain from [15, Cor. 2.2, Chap. II] the following lemma.

Lemma 4.5. Let $2 \leq q<\infty$. Then $-\Delta_{q}: W_{q}^{1}(\Omega) \rightarrow W_{q^{\prime}, 0}^{-1}(\Omega)$ is maximal monotone and $\lambda-\Delta_{q}: W_{q}^{1}(\Omega) \rightarrow W_{q^{\prime}, 0}^{-1}(\Omega)$ is bijective with strongly continuous inverse for every $\lambda>0$.

Proof. The fact that $-\Delta_{q}$ is maximal monotone follows from [15, Prop. 2.2, Chap. II]. Moreover, $\lambda-\Delta_{q}$ is coercive as seen above. Hence [15, Cor. 2.2 and Lemma 2.1] yield that $\lambda-\Delta_{q}$ is onto. Since $\lambda-\Delta_{q}$ is strictly monotone, $\lambda-\Delta_{q}$ is a bijection.

Finally, if $f_{n} \rightarrow_{n \rightarrow \infty} f$ in $W_{q^{\prime}, 0}^{-1}(\Omega)$ and $\left(\lambda-\Delta_{q}\right) u_{n}=f_{n},\left(\lambda-\Delta_{q}\right) u=f$, then

$$
\lambda\left\|u_{n}-u\right\|_{L^{2}}^{2}-\left\langle\Delta_{q} u_{n}-\Delta_{q} u, u_{n}-u\right\rangle=\left\langle f_{n}-f, u_{n}-u\right\rangle \rightarrow_{n \rightarrow \infty} 0
$$

since $f_{n} \rightarrow_{n \rightarrow \infty} f$ strongly and $\left\|u_{n}\right\|_{L^{2}}+\left\|\nabla u_{n}\right\|_{L^{q}} \leq C$ because of (4.12) and the boundedness of $\left\|f_{n}\right\|_{W_{q^{\prime}, 0}^{-1}}$. Hence $u_{n} \rightarrow_{n \rightarrow \infty} u$ in $L^{2}(\Omega)$. Since $u_{n}, n \in \mathbb{N}$, is bounded in $W_{q}^{1}(\Omega), u_{n} \rightarrow_{n \rightarrow \infty} u$ in $W_{q}^{1}(\Omega)$. Finally, since

$$
\lim _{n \rightarrow \infty}\left\|\nabla u_{n}\right\|_{L^{q}}^{q}=\lim _{n \rightarrow \infty}-\left\langle\Delta_{q} u_{n}, u_{n}\right\rangle=-\left\langle\Delta_{q} u, u\right\rangle=\|\nabla u\|_{L^{q}}^{q},
$$

$u_{n} \rightarrow_{n \rightarrow \infty} u$ strongly in $W_{q}^{1}(\Omega)$.

Remark 4.6. If we consider $-\Delta_{q}: L^{q}\left(0, T ; W_{q}^{1}(\Omega)\right) \rightarrow L^{q^{\prime}}\left(0, T ; W_{q^{\prime}, 0}^{1}(\Omega)\right)$, then $-\Delta_{q}$ is still maximal monotone since

$$
\int_{0}^{T}\left\langle-\Delta_{q} u-f, u-v\right\rangle_{W_{q^{\prime}, 0}^{-1}, W_{q}^{1}} d t \geq 0
$$


for all $u \in L^{q}\left(0, T ; W_{q}^{1}(\Omega)\right)$ and some $f \in L^{q^{\prime}}\left(0, T ; W_{q^{\prime}, 0}^{1}(\Omega)\right), v \in L^{q}\left(0, T ; W_{q}^{1}(\Omega)\right)$ implies $-\Delta_{q} v(t)=f(t)$ for almost all $t \in(0, T)$, which can be seen as follows: Choosing $u=v+t w$ above with $w \in L^{q}\left(0, T ; W_{q}^{1}(\Omega)\right), t \in \mathbb{R}$, arbitrary, we obtain

$$
t \int_{0}^{T}\left\langle-\Delta_{q}(v+t w)-f, w\right\rangle_{W_{q^{\prime}, 0}^{-1}, W_{q}^{1}} d t \geq 0 .
$$

Dividing by $t$ and passing $t \rightarrow 0$, we obtain

$$
\int_{0}^{T}\left\langle-\Delta_{q} v-f, w\right\rangle_{W_{q^{\prime}, 0}^{-1}, W_{q}^{1}} d t \geq 0
$$

for all $w \in L^{q}\left(0, T ; W_{q}^{1}(\Omega)\right)$, which implies $-\Delta_{q} v=f$ almost everywhere.

\section{Approximate System and Implicit Time Discretization}

In this section we construct weak solutions of the approximate system

$$
\begin{gathered}
\rho \partial_{t} v+\rho v \cdot \nabla v-\operatorname{div} S(c, D v)+\rho \nabla g_{0}-\delta g_{0} \frac{v}{2}=\rho \mu_{0} \nabla c \text { in } Q, \\
\rho_{t}+\operatorname{div}(\rho v)+\delta g_{0}=0 \text { in } Q, \\
P_{0}\left(\rho c_{t}+\rho v \cdot \nabla c\right)=\operatorname{div}\left(m(c) \nabla \mu_{0}\right) \text { in } Q,
\end{gathered}
$$

and

$$
\rho \mu_{0}+\rho^{2} \bar{p}(t)=\beta \rho^{2} g_{0}+\phi(c)-\frac{\partial A}{\partial c} \Delta_{q} A(c),
$$

together with (1.5)-(1.9). Here as before $P_{0} f=f-\bar{f}$, where $\bar{f}$ denotes the mean value of $f$ on $\Omega$. Because of the damping term $\delta g_{0}$ in (5.2), every sufficiently smooth solution of the latter system satisfies

$$
\begin{aligned}
& \frac{d}{d t} E(c(t), v(t))=-\int_{\Omega} S(c(t), D v(t)): D v(t) d x \\
& \quad-\int_{\Omega} m(c)|\nabla \mu|^{2} d x-\gamma \int_{\partial \Omega}|v|^{2} d \sigma-\delta \int_{\Omega}\left|g_{0}\right|^{2} d x,
\end{aligned}
$$

which implies an additional $L^{2}(Q)$-bound of $g_{0}$. The latter energy identity can be obtained by multiplying (5.1) by $v,(5.3)$ by $\mu,(5.4)$ by $\partial_{t} c$ and using (5.2) together with $-\beta \rho^{2} \partial_{t} c=\partial_{t} \rho$. Similar calculations are contained in the following proofs.

The main result of this section is:

Theorem 5.1. Let $q>d, d=2,3, \delta>0$, let Assumption 2.1 be satisfied and let $R, \varepsilon>0$ and $\Phi \in C^{2}(\mathbb{R}), \Phi(s) \geq 0, \Phi^{\prime \prime}(s) \geq-M$ be such that (2.7) holds. Then for every $v_{0} \in L^{2}(\Omega)^{d}, c_{0} \in W_{q}^{1}(\Omega)$ with $E\left(v_{0}, c_{0}\right)<R$, there are some

$$
\begin{aligned}
v & \in B C_{w}\left([0, \infty) ; L^{2}(\Omega)^{d}\right) \cap L^{2}\left(0, \infty ; H_{n}^{1}(\Omega)\right), \\
c & \in B C_{w}\left([0, \infty) ; W_{q}^{1}(\Omega)\right), \quad \mu_{0} \in L^{2}\left(0, \infty ; H_{(0)}^{1}(\Omega)\right), \\
g_{0} & \in L^{2}\left(0, \infty ; L_{(0)}^{2}(\Omega)\right), \quad \bar{p} \in L_{\mathrm{uloc}}^{2}([0, \infty))
\end{aligned}
$$

with $\left.(v, c)\right|_{t=0}=\left(v_{0}, c_{0}\right)$ solving (5.1)-(5.4) together with (1.6)-(1.8) in the following weak sense: 
1. For every $\psi \in C_{0}^{\infty}\left(0, \infty ; H_{n}^{1}(\Omega) \cap L^{\infty}(\Omega)^{d}\right)$,

$$
\begin{gathered}
-\left(v, \partial_{t} \psi\right)_{Q}+(v \cdot \nabla v, \psi)_{Q}+\left(\rho^{-1} S(c, D v), \nabla \psi\right)_{Q}+\left(S(c, D v), \nabla \rho^{-1} \otimes \psi\right)_{Q} \\
+\gamma\left(\rho^{-1} v, \psi\right)_{S}-\left(g_{0}, \operatorname{div} \psi\right)_{Q}-\frac{\delta}{2}\left(\rho^{-1} g_{0} v, \psi\right)_{Q}=\left(\mu_{0} \nabla c, \psi\right)_{Q} .
\end{gathered}
$$

2. For every $\varphi \in C_{0}^{\infty}\left(0, \infty ; C^{1}(\bar{\Omega})\right)$,

$$
\begin{gathered}
-\left(\rho, \partial_{t} \varphi\right)_{Q}-(\rho v, \nabla \varphi)_{Q}+\delta\left(g_{0}, \varphi\right)_{Q}=0 \\
\left(\rho c, P_{0} \partial_{t} \varphi\right)_{Q}+(\rho c v, \nabla \varphi)_{Q}-\delta\left(g_{0} c, P_{0} \varphi\right)_{Q}=\left(m(c) \nabla \mu_{0}, \nabla \varphi\right)_{Q}, \\
\left(a^{-\frac{1}{q}}(c)\left(\rho \mu_{0}+\rho^{2}\left(\bar{p}-\beta g_{0}\right)-\phi(c)\right), \varphi\right)_{Q}=\left(|\nabla \Lambda|^{q-2} \nabla \Lambda, \nabla \varphi\right)_{Q},
\end{gathered}
$$

where $A=A(c)$.

\section{Moreover,}

$$
\begin{aligned}
& E(c(t), v(t))+\gamma\|v\|_{L^{2}\left(S_{(s, t)}\right)}^{2}+\delta\left\|g_{0}\right\|_{L^{2}\left(Q_{(s, t)}\right)}^{2} \\
& \quad+\int_{Q_{(s, t)}}\left(S(c(t), D v(t)): D v(t)+m(c)\left|\nabla \mu_{0}\right|^{2}\right) d(x, \tau) \leq E(c(s), v(s))
\end{aligned}
$$

for all $t \in[s, \infty)$ and almost all $s \in[0, \infty)$ including $s=0$.

We note that for the weak formulation (5.7) one uses that (5.2) implies

$$
\rho \partial_{t} c+\rho v \cdot \nabla c=\partial_{t}(\rho c)+\operatorname{div}(\rho c v)+\delta g_{0} c .
$$

In order to prove the latter theorem, we use an implicit time discretization. To this end, let $h=\frac{1}{N}, N \in \mathbb{N}$. Then for given $v_{k}, c_{k}$ and $\rho_{k}=\hat{\rho}\left(c_{k}\right), k \in \mathbb{N}_{0}$, we determine $\left(v_{k+1}, g_{0, k+1}, c_{k+1}, \mu_{0, k+1}, \bar{p}_{k+1}\right)$ as a solution of the non-linear elliptic system

$$
\begin{aligned}
& \left(\rho_{k} \frac{v-v_{k}}{h}+\rho_{k} v \cdot \nabla v, \psi\right)_{\Omega}+\left(S\left(c_{k}, D v\right), \nabla \psi\right)_{\Omega} \\
& \quad+\gamma(v, \psi)_{\partial \Omega}+\left(g_{0}, \operatorname{div}\left(\rho_{k} \psi\right)\right)_{\Omega}=\left(\rho_{k} \mu_{0} \nabla c, \psi\right)_{\Omega}+\frac{\delta}{2}\left(g_{0} v, \psi\right)_{\Omega}
\end{aligned}
$$

for all $\psi \in H_{n}^{1}(\Omega)$,

$$
\begin{aligned}
\frac{\rho-\rho_{k}}{h}+\operatorname{div}\left(\rho_{k} v\right)+\delta g_{0} & =0 \quad \text { in } \Omega \\
\left(\rho_{k} \frac{c-c_{k}}{h}+\rho_{k} v \cdot \nabla c, \varphi\right)_{\Omega} & =-\left(m\left(c_{k}\right) \nabla \mu_{0}, \nabla \varphi\right)_{\Omega}
\end{aligned}
$$

for all $\varphi \in H_{(0)}^{1}(\Omega)$, and

$$
\begin{aligned}
& \left(|\nabla A(c)|^{q-2} \nabla A(c), \nabla \varphi\right)_{\Omega} \\
& =\left(\frac{c-c_{k}}{A(c)-A\left(c_{k}\right)}\left(\rho_{k} \mu_{0}+\rho \rho_{k} \bar{p}-\beta \rho \rho_{k} g_{0}-\phi_{0}(c)+\kappa \frac{c+c_{k}}{2}\right), \varphi\right)_{\Omega}
\end{aligned}
$$


for all $\varphi \in W_{q}^{1}(\Omega)$. Here $\rho=\hat{\rho}(c), \rho_{k}=\hat{\rho}\left(c_{k}\right)$, and $\kappa \in \mathbb{R}$ is chosen such that $\Phi(c)=\Phi_{0}(c)-\frac{\kappa}{2} c^{2}$ with $\Phi_{0}(c)$ convex, which is possible since $\Phi^{\prime \prime}(c) \geq-M>$ $-\infty$. Moreover, we note that, if $c=c_{k}$, then $\frac{c-c_{k}}{A(c)-A\left(c_{k}\right)}$ above has to be replaced by $A^{\prime}(c)^{-1}=a^{-\frac{1}{q}}(c)$. For simplicity, we will write $\frac{c-c_{k}}{A(c)-A\left(c_{k}\right)}$ even in the case $c=c_{k}$, having the latter replacement in mind.

Finally, we note that (5.11) implies

$$
\int_{\Omega} \rho d x=\int_{\Omega} \rho_{k} d x .
$$

Lemma 5.2. Let $q>d=2,3$, let $R, h, \delta>0,0<\varepsilon<\frac{|\alpha|}{|\beta|}-1$, and let $\Phi \in C^{2}(\mathbb{R}), \Phi(c) \geq 0, \Phi^{\prime \prime}(c) \geq-M$ be chosen such that (2.7) holds. Then for every $\left(v_{k}, c_{k}\right) \in L^{2}(\Omega)^{d} \times W_{q}^{1}(\Omega)$ with $E\left(v_{k}, c_{k}\right)<R$ there are some

$$
\left(v, g_{0}, c, \mu_{0}, \bar{p}\right) \in H_{n}^{1}(\Omega) \times L_{(0)}^{2}(\Omega) \times W_{q}^{1}(\Omega) \times H^{2}(\Omega) \cap L_{(0)}^{2}(\Omega) \times \mathbb{R}
$$

solving (5.10)-(5.13) and which satisfy the discrete energy estimate

$$
\begin{aligned}
& E(c, v)+\int_{\Omega} \rho_{k} \frac{\left|v-v_{k}\right|^{2}}{2} d x+\delta h\left\|g_{0}\right\|_{L^{2}(\Omega)}^{2}+\gamma h\|v\|_{L^{2}(\partial \Omega)}^{2} \\
& \quad+h \int_{\Omega} S\left(c_{k}, D v\right): D v d x+h \int_{\Omega} m\left(c_{k}\right)\left|\nabla \mu_{0}\right|^{2} d x \leq E\left(c_{k}, v_{k}\right) .
\end{aligned}
$$

Moreover, there is a constant $C(R)$ independent of $\left(v_{k}, c_{k}\right)$ such that $|\bar{p}| \leq c$.

Proof. We first show the a priori estimate (5.15) for any $\left(v, g_{0}, c, \mu, \bar{p}\right) \in H^{1}(\Omega)^{d} \times$ $L^{2}(\Omega) \times W_{q}^{1}(\Omega) \times H^{2}(\Omega) \times \mathbb{R}$ solving (5.10)-(5.13) and satisfying $E_{\text {free }}(c) \leq R$. First of all, because of (5.11) multiplied with $\frac{1}{2}|v|^{2}$ we obtain

$$
\begin{aligned}
\int_{\Omega} \rho_{k}(v \cdot \nabla v) \cdot v d x & =\int_{\Omega} \rho_{k} v \cdot \nabla \frac{|v|^{2}}{2} d x=-\int_{\Omega} \operatorname{div}\left(\rho_{k} v\right) \frac{|v|^{2}}{2} \\
& =\int_{\Omega} \frac{\rho-\rho_{k}}{h} \frac{|v|^{2}}{2}+\delta \int_{\Omega} g_{0} \frac{|v|^{2}}{2} .
\end{aligned}
$$

Thus

$$
\begin{aligned}
& \int_{\Omega} \frac{\left(\rho_{k}|v|^{2}-\rho_{k} v_{k} \cdot v\right)}{h} d x+\int_{\Omega} \rho_{k}(v \cdot \nabla v) \cdot v d x-\delta \int_{\Omega} g_{0} \frac{|v|^{2}}{2} d x \\
& \quad=\int_{\Omega} \rho_{k} \frac{|v|^{2}}{2 h} d x-\int_{\Omega} \rho_{k} \frac{\left|v_{k}\right|^{2}}{2 h} d x+\int_{\Omega} \rho_{k} \frac{\left|v-v_{k}\right|^{2}}{2 h} d x+\int_{\Omega} \frac{\rho-\rho_{k}}{h} \frac{|v|^{2}}{2} d x \\
& =\int_{\Omega} \rho \frac{|v|^{2}}{2 h} d x-\int_{\Omega} \rho_{k} \frac{\left|v_{k}\right|^{2}}{2 h} d x+\int_{\Omega} \rho_{k} \frac{\left|v-v_{k}\right|^{2}}{2 h} d x
\end{aligned}
$$

where we have used the simple algebraic relation

$$
a \cdot(a-b)=\frac{|a|^{2}}{2}-\frac{|b|^{2}}{2}+\frac{|a-b|^{2}}{2}, \quad a, b \in \mathbb{R}^{d} .
$$


Hence, choosing $\psi=v \in H_{n}^{1}(\Omega)$ in (5.10), we derive

$$
\begin{gathered}
\int_{\Omega} \rho \frac{|v|^{2}}{2 h} d x+\int_{\Omega} \rho_{k} \frac{\left|v-v_{k}\right|^{2}}{2 h} d x+\int_{\Omega} S\left(c_{k}, D v\right): D v d x+\gamma \int_{\partial \Omega}|v|^{2} d \sigma \\
=\int_{\Omega} \rho_{k} \frac{\left|v_{k}\right|^{2}}{2 h} d x+\int_{\Omega} \rho_{k} \mu_{0} \nabla c \cdot v d x+\int_{\Omega} g_{0} \operatorname{div}\left(\rho_{k} v\right) d x .
\end{gathered}
$$

Moreover, choosing $\varphi=\mu_{0}$ in (5.12), we conclude

$$
\int_{\Omega} \rho_{k} \frac{c-c_{k}}{h} \mu_{0} d x+\int_{\Omega} \rho_{k} \mu_{0} \nabla c \cdot v d x=-\int_{\Omega} m\left(c_{k}\right)\left|\nabla \mu_{0}\right|^{2} d x,
$$

where

$$
\begin{aligned}
& \int_{\Omega} \rho_{k} \frac{c-c_{k}}{h} \mu_{0} d x+\int_{\Omega} \rho \rho_{k} \frac{c-c_{k}}{h} d x \bar{p}=\int_{\Omega} \phi_{0}(c) \frac{c-c_{k}}{h} d x-\kappa \int_{\Omega} \frac{c^{2}-c_{k}^{2}}{h} d x \\
& \quad+\frac{1}{h} \int_{\Omega}|\nabla A(c)|^{q-2} \nabla A(c) \cdot \nabla\left(A(c)-A\left(c_{k}\right)\right) d x-\int_{\Omega} \frac{\rho-\rho_{k}}{h} g_{0} d x \\
& \geq \frac{1}{h}\left(E_{\text {free }}(c)-E_{\text {free }}\left(c_{k}\right)\right)+\int_{\Omega} g_{0} \operatorname{div}\left(\rho_{k} v\right) d x
\end{aligned}
$$

because of (5.13) with $\varphi=\frac{1}{h}\left(A(c)-A\left(c_{k}\right)\right)$. Here we have used (5.11), (5.16), the simple relation

$$
\rho-\rho_{k}=\beta \rho \rho_{k}\left(c_{k}-c\right),
$$

and that

$$
\begin{aligned}
\phi_{0}(c)\left(c-c_{k}\right) & \geq \Phi_{0}(c)-\Phi_{0}\left(c_{k}\right),\left(|\nabla u|^{q-2} \nabla u, \nabla\left(u-u_{k}\right)\right)_{\Omega} \\
& \geq \frac{1}{q}\left(\|\nabla u\|_{L^{q}}^{q}-\left\|\nabla u_{k}\right\|_{L^{q}}^{q}\right),
\end{aligned}
$$

since $\Phi_{0}$ is convex, $-\Delta_{q} u$ is the subgradient of $u \mapsto \frac{1}{q}\|\nabla u\|_{L^{q}(\Omega)}^{q}$, cf. Sect. 4. Moreover, because of (5.20) and (5.14),

$$
-\beta \int_{\Omega} \rho \rho_{k} \frac{c-c_{k}}{h} d x \bar{p}=\int_{\Omega}\left(\rho-\rho_{k}\right) d x \bar{p}=0 .
$$

Combining (5.17)-(5.19), we conclude (5.15).

Furthermore, we note that, since $c(x) \in[-1-\varepsilon, 1+\varepsilon]$ due to $E_{\text {free }}(c) \leq R$ by assumption and (2.7), we conclude

$$
\|c\|_{W_{q}^{1}(\Omega)} \leq C(\varepsilon, R) .
$$

Finally, the estimate $|\bar{p}| \leq C$ follows from (5.13) with $\varphi=\frac{A(c)-A\left(c_{k}\right)}{c-c_{k}} \rho^{-1} \rho_{k}^{-1}$.

In order to show existence of weak solutions, we use a homotopy argument based on the Leray-Schauder degree, cf. e.g. [8]. To this end we introduce the operators $\mathcal{L}_{k}, \mathcal{F}_{k}: X \rightarrow Y$ where

$$
\begin{aligned}
& X=H_{n}^{1}(\Omega) \times L_{(0)}^{2}(\Omega) \times W_{q}^{1}(\Omega) \times H_{(0)}^{1}(\Omega) \times \mathbb{R}, \\
& Y=H_{n}^{-1}(\Omega) \times L_{(0)}^{2}(\Omega) \times H_{(0)}^{-1}(\Omega) \times W_{q^{\prime}, 0}^{-1}(\Omega),
\end{aligned}
$$


and for $w=\left(v, g_{0}, c, \mu_{0}, \bar{p}\right) \in X$,

$$
\begin{aligned}
& \mathcal{L}_{k} w=\left(\begin{array}{c}
L_{k}\left(v, g_{0}\right) \\
\operatorname{div}\left(\rho_{k} v\right)+\delta g_{0}+\bar{p} \\
\operatorname{div}_{n}\left(m\left(c_{k}\right) \nabla \mu_{0}\right) \\
A(c)-\Delta_{q} A(c)
\end{array}\right), \\
&\left\langle L_{k}\left(v, g_{0}\right), \varphi\right\rangle_{H_{n}^{-1}, H_{n}^{1}}=\left(S\left(c_{k}, D v\right), \nabla \varphi\right)_{\Omega}+\gamma(v, \varphi)_{\partial \Omega}-\left(g_{0}, \operatorname{div}\left(\rho_{k} \varphi\right)\right)_{\Omega}
\end{aligned}
$$

for all $\varphi \in H_{n}^{1}(\Omega)$ and

$$
\begin{aligned}
\mathcal{F}_{k} w & =\left(\begin{array}{c}
\rho_{k} \mu_{0} \nabla c+\frac{\delta}{2} g_{0} v-\rho_{k} \frac{v-v_{k}}{h}-\rho_{k} v \cdot \nabla v \\
-P_{0}\left(\frac{\hat{\rho}(c)-\rho_{k}}{h}\right)-\int_{\Omega} \hat{\rho}(c) \rho_{k} \frac{c-c_{k}}{h} d x+\bar{p} \\
P_{0}\left(\rho_{k} \frac{c-c_{k}}{h}+\rho_{k} v \cdot \nabla c\right) \\
\frac{c-c_{k}}{A(c)-A\left(c_{k}\right)} F_{k}\left(g_{0}, c, \mu_{0}, \bar{p}\right)+A(c)
\end{array}\right), \quad \text { where } \\
F_{k}\left(c, g_{0}, \mu\right) & =\rho_{k} \mu_{0}+\rho_{k} \rho \bar{p}-\beta \rho \rho_{k} g_{0}-\phi_{0}(c)+\kappa \frac{c+c_{k}}{2} .
\end{aligned}
$$

Note that $\operatorname{div}_{n}: L^{2}(\Omega)^{d} \rightarrow H_{(0)}^{-1}(\Omega)$ is defined as in (4.3). Here we have to modify $\hat{\rho}(c)=\frac{1}{\alpha+\beta c}$ outside of $[-1-\varepsilon, 1+\varepsilon]$ to some $\hat{\rho} \in C^{1}(\mathbb{R})$ in order to have $\mathcal{F}_{k}(w)$ well defined for all $c \in W_{q}^{1}(\Omega)$.

Using the lemma of Lax-Milgram,

$$
\left(\begin{array}{c}
L_{k}\left(v, g_{0}\right) \\
\operatorname{div}\left(\rho_{k} v\right)+\delta g_{0}
\end{array}\right)=\left(\begin{array}{l}
f_{1} \\
f_{2}
\end{array}\right) \in \begin{gathered}
H_{n}^{-1}(\Omega) \\
L_{(0)}^{2}(\Omega)
\end{gathered}
$$

has a unique solution $v \in H_{n}^{1}(\Omega), g_{0} \in L_{(0)}^{2}(\Omega)$ since

$$
\left\langle L_{k}\left(v, g_{0}\right), v\right\rangle_{H_{n}^{1}, H_{n}^{-1}}+\left(\operatorname{div}\left(\rho_{k} v\right), g_{0}\right)_{\Omega}+\delta\left(g_{0}, g_{0}\right)_{\Omega} \geq c_{0}\|v\|_{H^{1}(\Omega)}^{2}+\delta\left\|g_{0}\right\|_{L^{2}(\Omega)}^{2} .
$$

Moreover, we note that $\operatorname{div}\left(\rho_{k} v\right)+\bar{p}=f \in L^{2}(\Omega)$ is equivalent to $\operatorname{div}\left(\rho_{k} v\right)=P_{0} f$ and $\bar{p}=m(f)$, since $\int_{\Omega} \operatorname{div}\left(\rho_{k} v\right) d x=0$ due to $\left.n \cdot v\right|_{\partial \Omega}=0$. The invertibility of $\operatorname{div}_{n}\left(m\left(c_{k}\right) \nabla \cdot\right): H_{(0)}^{1}(\Omega) \rightarrow H_{(0)}^{-1}(\Omega)$ is a simple consequence of the lemma of Lax-Milgram too, the invertibility of $I-\Delta_{q}: W_{q}^{1}(\Omega) \rightarrow W_{q^{\prime}, 0}^{-1}(\Omega)$ follows from Lemma 4.5, and $c \mapsto A(c)$ is an invertible mapping on $W_{q}^{1}(\Omega)$, since $A^{\prime}(c)=a^{\frac{1}{q}}(c) \geq$ $m_{0}^{\frac{1}{q}}>0$. Altogether it follows that $\mathcal{L}_{k}: X \rightarrow Y$ is invertible and $\mathcal{L}_{k}^{-1}: Y \rightarrow X$ is continuous.

Therefore $w=\left(v, g_{0}, c, \mu_{0}, \bar{p}\right)$ is a solution of (5.10)-(5.13) if and only if $\mathcal{L}_{k}(w)-$ $\mathcal{F}_{k}(w)=0$, where we note that $\mathcal{L}_{k}(w)=\mathcal{F}_{k}(w)$ implies

$$
0=\int_{\Omega} \hat{\rho}(c) \rho_{k}\left(c-c_{k}\right) d x=-\beta^{-1} \int_{\Omega}\left(\hat{\rho}(c)-\rho_{k}\right) d x
$$

due to (5.20). Moreover, $\mathcal{L}_{k}(w)-\mathcal{F}_{k}(w)=0$ is equivalent to

$$
w-\mathcal{L}_{k}^{-1}\left(\mathcal{F}_{k}(w)\right)=0
$$


since $\mathcal{L}_{k}^{-1}(0)=0$ due to $A(0)=0$. Moreover, it is easy to observe that $\mathcal{F}_{k}: X \rightarrow Y$ is a continuous and bounded mapping, where we note that

$$
W_{q}^{1}(\Omega) \ni c \mapsto \frac{c-c_{k}}{A(c)-A\left(c_{k}\right)} \in W_{q}^{1}(\Omega)
$$

is continuous since

$$
\frac{A(c)-A\left(c_{k}\right)}{c-c_{k}}=\int_{0}^{1} A^{\prime}\left(\tau c+(1-\tau) c_{k}\right) d \tau=\int_{0}^{1} a^{\frac{1}{q}}\left(\tau c+(1-\tau) c_{k}\right) d \tau
$$

and $a(s) \geq m_{0}>0$. Moreover, $\mathcal{K}_{k}(w):=\mathcal{L}_{k}^{-1}\left(\mathcal{F}_{k}(w)\right)$ defines a compact operator on $X$ since

$$
\mathcal{F}_{k}(w) \in L^{\frac{3}{2}}(\Omega)^{d} \times W_{q}^{1}(\Omega) \times L_{(0)}^{2}(\Omega) \times L^{2}(\Omega) \hookrightarrow \hookrightarrow Y
$$

for all $w \in X$.

Now we are able to apply a homotopy argument in order to show that the LeraySchauder degree of $I-\mathcal{K}_{k}$ at 0 is 1 in some suitable open set $U$. To this end, let $\hat{\rho}_{\tau}(c)=(\alpha+(1-\tau) \beta c)^{-1}, \tau \in[0,1]$. Replacing $v_{k}, c_{k}, \rho, \rho_{k}, \beta, \kappa$, and $\phi_{0}$ in $(5.10)-$ (5.13) by

$$
\begin{aligned}
& v_{k}^{\tau}=(1-\tau) v_{k}, \quad c_{k}^{\tau}=(1-\tau) c_{k}, \quad \rho_{\tau}=\hat{\rho}_{\tau}(c), \quad \rho_{k}^{\tau}=\hat{\rho}_{\tau}\left(c_{k}^{\tau}\right), \\
& \beta_{\tau}=(1-\tau) \beta, \quad \kappa_{\tau}=(1-\tau) \kappa, \quad \phi_{0}^{\tau}(s)=(1-\tau) \phi_{0}(s)
\end{aligned}
$$

for every $\tau \in[0,1]$, and denoting by $\mathcal{L}_{k}^{\tau}, \mathcal{F}_{k}^{\tau}, \mathcal{K}_{k}^{\tau}$ the corresponding operators, we get a family of compact operator $\mathcal{K}_{k}^{\tau}$, depending continuously on $\tau \in[0,1]$ such that $\mathcal{K}_{k}^{0}=\mathcal{K}_{k}$ and

$$
\operatorname{deg}\left(I+\mathcal{K}_{k}^{0}, 0, U\right)=\operatorname{deg}\left(I+\mathcal{K}_{k}^{1}, 0, U\right)
$$

provided that

$$
\left(I+\mathcal{K}_{k}^{\tau}\right)(w) \neq 0 \quad \text { for all } w \in \partial U, \tau \in[0,1] .
$$

Because of the energy estimate (5.15) and the estimate of $\bar{p}$, we have $\|w\|_{X}<C(R, \delta)$ for any solution of (5.10)-(5.13) such that $E_{\text {free }}(c) \leq R$, which implies the latter condition for $U=\left\{w \in X:\|w\|_{X}<C(R, \delta), E_{\text {free }}(c)<R\right\}$.

In order to show that $\operatorname{deg}\left(I+\mathcal{K}_{k}^{1}, 0, U\right)=1$, we define a second homotopy by

$$
\mathcal{K}_{k}^{\tau}(w)=\left(\mathcal{L}_{k}^{1}\right)^{-1}(2-\tau) \mathcal{F}_{k}^{1}(w), \quad \tau \in[1,2] .
$$

Then $w-\mathcal{K}_{k}^{\tau}(w)=0$ if and only if $w=\left(v, g_{0}, c, \mu_{0}, \bar{p}\right)$ is a solution of

$$
\begin{aligned}
\lambda\left(\rho_{0} \frac{v}{h}+\rho_{0} v \cdot \nabla v-\frac{\delta}{2} g_{0} v, \varphi\right)_{\Omega} & +(v(0) D v, D \varphi)_{\Omega}+\gamma(v, \varphi)_{\partial \Omega}, \\
& =\left(\rho_{0} g_{0}, \operatorname{div} \varphi\right)_{\Omega}+\lambda\left(\rho_{0} \mu_{0} \nabla c, \varphi\right)_{\Omega}, \\
\rho_{0} \operatorname{div} v+\delta g_{0} & =0 \\
\lambda \rho_{0} \frac{c}{h}+\lambda \rho_{0} v \cdot \nabla c & =\operatorname{div}\left(m(0) \nabla \mu_{0}\right), \\
(1-\lambda) A(c)-\Delta_{q} A(c) & =\lambda \frac{c}{A(c)}\left(\rho_{0} \mu_{0}+\rho_{0}^{2} \bar{p}\right), \\
(1-\lambda) \bar{p} & =-\rho_{0}^{2} \int_{\Omega} \frac{c}{h} d x
\end{aligned}
$$


for all $\varphi \in H_{n}^{1}(\Omega)$ together with $\left.n \cdot v\right|_{\partial \Omega}=\left.\partial_{n} c\right|_{\partial \Omega}=\left.\partial_{n} \mu_{0}\right|_{\partial \Omega}=0$, where $\rho_{0}=\hat{\rho}(0)=$ $\alpha^{-1}$ and $\lambda=(2-\tau)$. Choosing $\varphi=v$ in (5.22), multiplying (5.24) by $\mu_{0}$ and (5.25) by $\frac{A(c)}{h}$, we obtain by similar calculations as before

$$
\begin{aligned}
& \lambda \rho_{0} \frac{\|v\|_{2}^{2}}{2 h}+(1-\lambda) \frac{\|A(c)\|_{2}^{2}}{2 h}+\lambda \frac{\|\nabla A(c)\|_{q}^{q}}{q h}+(1-\lambda) \lambda \bar{p}^{2} \\
& \quad+v(0)\|D v\|_{2}^{2}+\gamma\|v\|_{L^{2}(\partial \Omega)}^{2}+\delta\left\|g_{0}\right\|_{2}^{2}+m(0)\left\|\nabla \mu_{0}\right\|_{2}^{2}=0,
\end{aligned}
$$

where one uses that

$$
\begin{aligned}
\int_{\Omega}\left(\rho_{0} v \cdot \nabla v \cdot v-\frac{\delta}{2} g_{0}|v|^{2}\right) d x & =-\int_{\Omega}\left(\rho_{0} \operatorname{div} v+\delta g_{0}\right) \frac{|v|^{2}}{2} d x=0, \\
\frac{\|\nabla A(c)\|_{q}^{q}}{q} & =\frac{\|\nabla A(c)\|_{q}^{q}}{q}-\frac{\|\nabla A(0)\|_{q}^{q}}{q} \\
& \leq-\left\langle\Delta_{q} A(c), A(c)-A(0)\right\rangle=-\left\langle\Delta_{q} A(c), A(c)\right\rangle, \\
\lambda \rho_{0}^{2} \int_{\Omega} \frac{c}{h} d x \bar{p} & =-\lambda(1-\lambda) \bar{p}^{2}
\end{aligned}
$$

because of (5.23), and since $-\Delta_{q}$ is the subgradient of $u \mapsto \frac{\|\nabla u\|_{q}^{q}}{q}$. Hence (5.27) implies that $w=\mathcal{K}_{k}^{\tau}(w), \tau \in[1,2]$, if and only if $w=0$. Therefore any solution of $w-\mathcal{K}_{k}^{\tau}(w)$ remains in $U$ and

$$
\operatorname{deg}\left(I+\mathcal{K}_{k}, 0, U\right)=\operatorname{deg}\left(I+\mathcal{K}_{k}^{2}, 0, U\right)=\operatorname{deg}(I, 0, U)=1
$$

since $\mathcal{K}_{k}^{2}=0$. Therefore (5.10)-(5.13) has a solution in $U$.

Now let $N \in \mathbb{N}$ be given and let $\left(v_{k+1}, g_{0, k+1}, c_{k+1}, \mu_{0, k+1}, \bar{p}_{k+1}\right), k \in \mathbb{N}_{0}:=\mathbb{N} \cup\{0\}$, be chosen successively as a solution of (5.10)-(5.13) with $h=\frac{1}{N}$ and $\left(v_{k}, c_{k}\right)$ as initial value. Moreover, define $f^{N}(t):[-h, \infty)$ by $f^{N}(t)=f_{k}$ for $t \in[(k-1) h, k h)$ and $f \in\left\{v, g_{0}, c, \mu_{0}\right\}$ (setting $g_{0,0}=\mu_{0,0}=0$ ). For the following we denote

$$
\begin{gathered}
\left(\Delta_{h}^{+} f\right)(t)=f(t+h)-f(t), \quad\left(\Delta_{h}^{-} f\right)(t)=f(t)-f(t-h), \\
g_{h} \equiv\left(\tau_{h}^{*} g\right)(t)=g(t-h), \partial_{t, h}^{ \pm} f=\frac{1}{h} \Delta_{h}^{ \pm} f .
\end{gathered}
$$

Then, choosing $\psi=\rho_{k}^{-1} \int_{k h}^{k(h+1)} \varphi(x, t) d t$ in (5.10), where $\varphi \in C_{0}^{\infty}\left((0, \infty) ; H_{n}^{1}(\Omega)\right)$, and summing over all $k \in \mathbb{N}_{0}$, gives

$$
\begin{aligned}
& \left(\partial_{t, h}^{-} v^{N}+v^{N} \cdot \nabla v^{N}, \varphi\right)_{Q}+\left(\widetilde{S}\left(c_{h}^{N}, D v^{N}\right)-g_{0}^{N} I, D \varphi\right)_{Q}+\gamma\left(\left(\rho_{h}^{N}\right)^{-1} v^{N}, \varphi\right)_{S} \\
& \quad-\frac{\delta}{2}\left(\left(\rho_{h}^{N}\right)^{-1} g_{0}^{N} v^{N}, \varphi\right)_{Q}=\left(\mu_{0}^{N} \nabla c^{N}, \varphi\right)_{Q}-\left(\nabla\left(\rho_{h}^{N}\right)^{-1} \cdot S\left(c_{h}^{N}, D v^{N}\right), \varphi\right)_{Q}
\end{aligned}
$$

where $\varphi \in C_{0}^{\infty}\left((0, \infty) ; H_{n}^{1}(\Omega)\right)$ is arbitrary and $\widetilde{S}(c, D v)=\hat{\rho}^{-1}(c) S(c, D v)$. Moreover, since

$$
\left(\partial_{t, h}^{-} v_{N}, \varphi\right)_{Q}=-\left(v_{N}, \partial_{t, h}^{+} \varphi\right)_{Q}+\left(v_{0},\left.\varphi\right|_{t=0}\right)_{\Omega}
$$


we conclude

$$
\begin{gathered}
-\left(v^{N}, \partial_{t, h}^{+} \varphi\right)_{Q}+\left(v^{N} \cdot \nabla v^{N}, \varphi\right)_{Q}+\left(\left(\widetilde{S}\left(c_{h}^{N}, D v^{N}\right)-g^{N} I, D \varphi\right)_{Q}+\gamma\left(\left(\rho_{h}^{N}\right)^{-1} v^{N}, \varphi\right)_{S}\right. \\
-\frac{\delta}{2}\left(\left(\rho_{h}^{N}\right)^{-1} g_{0}^{N} v^{N}, \varphi\right)_{Q}=\left(\mu_{0}^{N} \nabla c^{N}, \varphi\right)_{Q}-\left(\nabla\left(\rho_{h}^{N}\right)^{-1} \cdot S\left(c_{h}^{N}, D v^{N}\right), \varphi\right)_{Q},
\end{gathered}
$$

for all $\varphi \in C_{0}^{\infty}\left(0, \infty ; H_{n}^{1}(\Omega)\right)$. In the same way, one obtains that

$$
\begin{aligned}
-\left(\rho^{N}, \partial_{t, h}^{+} \psi\right)_{Q}-\left(\rho_{h}^{N} v^{N}, \nabla \psi\right)_{Q}+\delta\left(g_{0}, \psi\right) & =0, \\
\left(\rho^{N} c^{N}, \partial_{t, h}^{+} \psi\right)_{Q}+\left(\rho_{h}^{N} c^{N} v^{N}-m^{N} \nabla \mu^{N}, \nabla \psi\right)_{Q} & =\delta\left(g_{0}^{N} c^{N}, \psi\right)_{Q}
\end{aligned}
$$

for all $\psi \in C_{0}^{\infty}\left(0, \infty ; H_{(0)}^{1}(\Omega)\right)$, where $m^{N}=m\left(\tau_{h}^{*} c^{N}\right)$ and

$$
\begin{aligned}
& \left(\left|\nabla A\left(c^{N}\right)\right|^{q-2} \nabla A\left(c^{N}\right), \nabla \psi\right)_{Q} \\
& =\left(\frac{\Delta_{h}^{-} c^{N}}{\Delta_{h}^{-} A\left(c^{N}\right)}\left(\rho_{h}^{N} \mu_{0}^{N}+\rho^{N} \rho_{h}^{N}\left(\bar{p}^{N}-\beta g_{0}^{N}\right)-\phi_{0}\left(c^{N}\right)+\frac{\kappa}{2}\left(c^{N}+c_{h}^{N}\right)\right), \psi\right)_{Q}
\end{aligned}
$$

for all $\psi \in C_{0}^{\infty}\left(0, \infty ; W_{q}^{1}(\Omega)\right)$. Here we have used that (5.11) implies

$$
\rho_{k} \frac{c-c_{k}}{h}+\rho_{k} v \cdot \nabla c=\frac{\rho c-\rho_{k} c_{k}}{h}+\operatorname{div}\left(\rho_{k} v c\right)+\delta g_{0} c .
$$

Finally, let $E_{N}(t)$ be the piecewise linear interpolation of $E\left(c_{k}, v_{k}\right)$ at $t_{k}=k h, k \in \mathbb{N}_{0}$, and let

$$
\begin{aligned}
D_{N}(t)= & \gamma\left\|v_{k+1}\right\|_{L^{2}(\partial \Omega)}^{2}+\delta\left\|g_{0, k+1}\right\|_{L^{2}(\Omega)}^{2} \\
& +\int_{\Omega}\left(S\left(c_{k}, D v_{k+1}\right): D v_{k+1}+m\left(c_{k}\right)\left|\nabla \mu_{0, k+1}\right|^{2}\right) d x \\
& \text { for all } t \in\left(t_{k}, t_{k+1}\right), k \in \mathbb{N}_{0} .
\end{aligned}
$$

Then (5.15) implies

$$
-\frac{d}{d t} E_{N}(t)=\frac{E\left(c_{k}, v_{k}\right)-E\left(c_{k+1}, v_{k+1}\right)}{h} \geq D_{N}(t) \text { for all } t \in\left(t_{k}, t_{k+1}\right), k \in \mathbb{N}_{0},
$$

and therefore

$$
E\left(c_{0}, v_{0}\right) \varphi(0)+\int_{0}^{\infty} E_{N}(t) \varphi^{\prime}(t) d t \geq \int_{0}^{\infty} D_{N}(t) \varphi(t) d t
$$

for all $\varphi \in W^{1}(0, \infty)$ with $\varphi \geq 0$. In particular, we have

$$
\begin{aligned}
& E\left(c^{N}(t), v^{N}(t)\right)+\int_{Q_{(s, t)}}\left(2 v\left(c_{h}^{N}\right)\left|D v^{N}\right|^{2}+\eta\left(c_{h}^{N}\right)\left|\operatorname{div} v^{N}\right|^{2}\right) d(x, \tau)+\delta\left\|g_{0}^{N}\right\|_{L^{2}\left(S_{(s, t)}\right)}^{2} \\
& \quad+\gamma\left\|v^{N}\right\|_{L^{2}\left(S_{(s, t)}\right)}^{2}+\int_{Q_{(s, t)}} m\left(c_{h}^{N}\right)\left|\nabla \mu_{0}^{N}\right|^{2} d(x, \tau) \leq E\left(c^{N}(s), v^{N}(s)\right)
\end{aligned}
$$

for all $0 \leq s \leq t<\infty$ with $s, t \in h \mathbb{N}_{0}$. 
Using the bounds given by the energy estimate above, we can pass to a subsequence, again denoted by $\left(v^{N}, g_{0}^{N}, c^{N}, \mu_{0}^{N}, \bar{p}^{N}\right)$, such that

$$
\begin{gathered}
\left(v^{N}, \mu_{0}^{N}, g_{0}^{N}, \bar{p}^{N}\right) \rightarrow_{N \rightarrow \infty}\left(v, \mu_{0}, g_{0}, \bar{p}\right) \text { in } L^{2}\left(0, \infty ; H^{1}(\Omega)^{d+1} \times L^{2}(\Omega) \times \mathbb{R}\right), \\
\left(v^{N}, c^{N}\right) \rightarrow_{N \rightarrow \infty}^{*}(v, c) \text { in } L^{\infty}\left(0, \infty ; L^{2}(\Omega)^{d} \times W_{q}^{1}(\Omega)\right) .
\end{gathered}
$$

In order to pass to the limit in all non-linearities, we show strong convergence of $v^{N}, c^{N}, \nabla A\left(c^{N}\right)$.

To this end, we define $\tilde{\rho}^{N}$ and $\tilde{v}^{N}$ as piecewise linear interpolation of $\rho^{N}\left(t_{k}\right), v^{N}\left(t_{k}\right)$, resp., where $t_{k}=k h, k \in \mathbb{N}_{0}$. More precisely, $\tilde{\rho}^{N}=\frac{1}{h} \chi_{[0, h]} *_{t} \rho^{N}$ and $\tilde{v}^{N}=\frac{1}{h} \chi_{[0, h]} *_{t}$ $v^{N}$, where the convolution is only taken with respect to the time variable $t$.

Then $\partial_{t} \tilde{v}^{N}=\partial_{t, h}^{-} v^{N}$ and

$$
\left\|\tilde{v}^{N}-v^{N}\right\|_{H^{-s}(\Omega)} \leq h\left\|\partial_{t} \tilde{v}^{N}\right\|_{H^{-s}(\Omega)}, \quad\left\|\tilde{\rho}^{N}-\rho^{N}\right\|_{H^{-s}(\Omega)} \leq h\left\|\partial_{t} \tilde{\rho}^{N}\right\|_{H^{-s}(\Omega)}
$$

for all $s \geq 0$. Because of (5.30), $\partial_{t} \tilde{v}^{N}$ is bounded in $L^{2}\left(0, \infty ; H^{-s}(\Omega)\right)$ for any $s>\frac{d}{2}$, where one uses that $v^{N} \cdot \nabla v^{N} \in L^{2}\left(0, \infty ; L^{1}(\Omega)\right), \mu_{0}^{N} \nabla c^{N} \in L^{2}(Q)$ and $\nabla\left(\rho_{h}^{N}\right)^{-1}$. $S\left(c_{h}^{N}, D v^{N}\right) \in L^{2}\left(0, \infty ; L^{1}(\Omega)\right)$ are bounded due to (5.36). Hence, applying the lemma of Aubin-Lions, we conclude that

$$
\tilde{v}^{N} \rightarrow_{N \rightarrow \infty} v \quad \text { in } L^{2}\left(0, T ; H^{s}(\Omega)\right),
$$

for all $0 \leq s<1,0<T<\infty$, and for a suitable subsequence. On the other hand, (5.37) implies

$$
\tilde{v}^{N}-v^{N} \rightarrow_{N \rightarrow 0} 0 \quad \text { in } L^{2}\left(0, \infty ; H^{-s}(\Omega)\right) \text { if } s>\frac{d}{2} .
$$

Since $\tilde{v}^{N}, v^{N}$ are bounded in $L^{\infty}\left(0, \infty ; L^{2}(\Omega)\right)$, we also obtain

$$
\tilde{v}^{N}-v^{N} \rightarrow_{N \rightarrow 0} 0 \quad \text { in } L^{p}\left(0, T ; H^{-s}(\Omega)\right)
$$

for any $1<p<\infty$ and $s, T>0$ by interpolation. Moreover, since $\tilde{v}^{N}, v^{N}$ are bounded in $L^{2}\left(0, \infty ; H^{1}(\Omega)\right)$, we conclude

$$
v^{N} \rightarrow_{N \rightarrow \infty} v \quad \text { in } L^{2}\left(0, T ; H^{s}(\Omega)\right) \text { for all } T>0,0 \leq s<1 .
$$

Finally, since $\tilde{v}^{N} \in L^{\infty}\left(0, \infty ; L^{2}(\Omega)\right)$ is bounded and $\tilde{v}^{N}$ converges weakly in

$$
H^{1}\left(0, \infty ; H^{-s}(\Omega)\right) \hookrightarrow B U C\left([0, \infty) ; H^{-s}(\Omega)\right)
$$

for $s>\frac{d}{2}$, and $\left.\tilde{v}^{N}\right|_{t=0}=v_{0}$, we conclude $v \in B C_{w}\left([0, \infty) ; L^{2}(\Omega)\right)$ and $\left.v\right|_{t=0}=v_{0}$, cf. Lemma 4.1.

Similarly, because of (5.32), $\partial_{t} \tilde{\rho}^{N}$ is bounded in $L_{\text {uloc }}^{2}\left([0, \infty) ; H^{-1}(\Omega)\right)$. On the other hand, $\tilde{\rho}^{N} \in L^{\infty}\left(0, \infty ; W_{q}^{1}(\Omega)\right)$ because of $c \in L^{\infty}\left(0, \infty ; W_{q}^{1}(\Omega)\right)$ and $c(t, x) \in$ $[-1-\varepsilon, 1+\varepsilon]$. Therefore the lemma of Aubin-Lions yields that for a suitable subsequence,

$$
\tilde{\rho}^{N} \rightarrow_{N \rightarrow \infty} \tilde{\rho} \quad \text { in } L^{2}\left(Q_{T}\right) \text { for all } T>0,
$$


and almost everywhere for some $\tilde{\rho} \in L^{\infty}(Q)$. Furthermore, by (5.37)

$$
\tilde{\rho}^{N}-\rho^{N} \rightarrow_{N \rightarrow \infty} 0 \quad \text { in } L_{\mathrm{uloc}}^{2}\left([0, \infty) ; H^{-1}(\Omega)\right) .
$$

As before we conclude that also $\rho^{N}=\hat{\rho}\left(c^{N}\right)$ converges strongly in $L^{2}\left(Q_{T}\right)$ for every $T>0$ and almost everywhere. Furthermore, since $\alpha+\beta c^{N}=\left(\rho^{N}\right)^{-1}$, also $c^{N}$ converges strongly in $L^{2}\left(Q_{T}\right), T>0$, and almost everywhere and we conclude $\tilde{\rho}=\hat{\rho}(c)$. Since $\tilde{\rho}^{N} \in H_{\mathrm{uloc}}^{1}\left([0, \infty) ; H^{-1}(\Omega)\right) \hookrightarrow B U C\left([0, \infty) ; H^{-1}(\Omega)\right)$ and $\tilde{\rho}^{N} \in L^{\infty}\left(0, \infty ; W_{q}^{1}(\Omega)\right)$ are bounded, $\rho \in B C_{w}\left([0, \infty) ; W_{q}^{1}(\Omega)\right)$ due to Lemma 4.1. Moreover, since $\rho^{N} \in$ $H^{1}\left(0, T ; H^{-1}(\Omega)\right), 0<T<\infty$, converges weakly, $\left.\rho\right|_{t=0}=\rho_{0}=\left.\lim _{N \rightarrow \infty} \rho^{N}\right|_{t=0}$ weakly in $H^{-1}(\Omega)$. Therefore also $c \in B C_{w}\left([0, \infty) ; W_{q}^{1}(\Omega)\right)$ and $\left.c\right|_{t=0}=c_{0}$.

Using these convergence results, it is easy to pass to the limit in Eqs. (5.32), (5.33) to obtain (5.6), (5.7).

Moreover, the right-hand side of (5.34) converges to

$$
f=a(c)^{-\frac{1}{q}}\left(\rho \mu_{0}+\rho^{2}\left(\bar{p}(t)-\beta g_{0}\right)-\phi(c)\right)
$$

weakly in $L^{2}\left(Q_{T}\right)$ for every $T<\infty$, where one can use (5.21) in order to pass to the limit in $\frac{c-c_{k}}{A(c)-A\left(c_{k}\right)}$.

Hence $\Delta_{q} A\left(c^{N}\right)$ converges weakly in $L^{2}\left(Q_{T}\right)$ to $f$ for every $T<\infty$ and therefore

$$
-\left\langle\Delta_{q} A\left(c^{N}\right), A\left(c^{N}\right)\right\rangle_{X_{T}, X_{T}^{\prime}}=\left(f^{N}, A\left(c^{N}\right)\right)_{Q_{T}} \rightarrow_{N \rightarrow \infty}(f, A(c))_{Q_{T}}
$$

with $X_{T}=L^{q}\left(0, T ; W_{q}^{1}(\Omega)\right)$, where we note that $-\Delta_{q}$ is a maximal monotone operator on $X_{T}$ due to Remark 4.6. Hence Lemma 4.4 implies $f=-\Delta_{q} A(c)$ and $A\left(c^{N}\right) \rightarrow A(c)$ strongly in $L^{q}\left(0, T ; W_{q}^{1}(\Omega)\right)$ for every $0<T<\infty$ because of

$$
\int_{0}^{T}\left\|\nabla A\left(c^{N}\right)\right\|_{L^{q}(\Omega)}^{q} d t \rightarrow_{N \rightarrow \infty} \int_{0}^{T}\|\nabla A(c)\|_{L^{q}(\Omega)}^{q} d t .
$$

Since $A^{-1} \in C^{1}(\mathbb{R}), c^{N}$ converges strongly in $L^{q}\left(0, T ; W_{q}^{1}(\Omega)\right)$ too. Hence $(5.8)$ holds and

$$
\nabla\left(\tau_{h}^{*} \rho^{N}\right)^{-1} \cdot S\left(c^{N}, D v^{N}\right) \rightarrow_{N \rightarrow \infty} \nabla \rho^{-1} \cdot S(c, D v) \quad \text { in } L^{1}\left(Q_{T}\right), T<\infty .
$$

Using the convergence results above, it is easy to show that (5.31) converges to (5.5).

Finally, since $v^{N}(t) \rightarrow_{N \rightarrow \infty} v(t)$ in $L^{2}(\Omega)$ and $c^{N}(t) \rightarrow_{N \rightarrow \infty} c$ in $W_{q}^{1}(\Omega)$ for almost every $t \in(0, \infty)$ (for a suitable subsequence),

$$
E_{N}(t) \rightarrow_{N \rightarrow \infty} E(c(t), v(t)) \quad \text { for almost all } t \in(0, \infty) .
$$

Moreover, by lower semi-continuity of norms and almost everywhere convergence of $c^{N}$ to $c$,

$$
\liminf _{N \rightarrow \infty} \int_{0}^{\infty} D_{N}(t) \varphi(t) d t \geq \int_{0}^{\infty} D(t) \varphi(t) d t
$$

for all $\varphi \in W_{1}^{1}(0, \infty)$ with $\varphi \geq 0$, where

$$
\begin{aligned}
D(t)= & \gamma\|v(t)\|_{L^{2}(\partial \Omega)}^{2}+\delta\left\|g_{0}(t)\right\|_{L^{2}(\Omega)}^{2} \\
& \left.+\left.\int_{\Omega}(2 v(c(t)) \mid D v(t))|+\eta(c(t))| \operatorname{div} v(t)\right|^{2}+m(c(t))\left|\nabla \mu_{0}(t)\right|^{2}\right) d x .
\end{aligned}
$$


Hence, passing to the limit in (5.35), we obtain

$$
E\left(c_{0}, v_{0}\right) \varphi(0)+\int_{0}^{\infty} E(c(t), v(t)) \varphi^{\prime}(t) d t \geq \int_{0}^{\infty} D(t) \varphi(t) d t
$$

for all $\varphi \in W_{1}^{1}(0, \infty)$ with $\varphi \geq 0$. Because of Lemma 4.3, this implies (5.9).

\section{Existence of Weak Solutions for the General System}

Let $R, \varepsilon, c_{0}, v_{0}$, and $\Phi$ be as in the assumptions of Theorem 2.4. Moreover, for $0<\delta \leq 1$ let $\left(v, g_{0}, c, \mu_{0}, \bar{p}\right) \equiv\left(v_{\delta}, g_{0, \delta}, c_{\delta}, \mu_{0, \delta}, \bar{p}_{\delta}\right)$ be a solution of (5.1)-(5.4) together with (1.5)-(1.9) due to Theorem 5.1.

In order to pass to the limit $\delta \rightarrow 0$, we need a good representation of $g_{0, \delta}$ and a suitable estimate of $\bar{p}(t)$. As in (1.12), we use the decomposition

$$
g_{0, \delta}=g_{1, \delta}-\partial_{t} G\left(v_{\delta}\right)
$$

where $G(v)=\Delta_{N}^{-1} \operatorname{div} v$ due to (1.13)-(1.14). Then (5.1) is equivalent to

$$
\partial_{t} P_{\sigma} v_{\delta}+v_{\delta} \cdot \nabla v_{\delta}-\rho_{\delta}^{-1} \operatorname{div} S\left(c_{\delta}, D v_{\delta}\right)+\nabla g_{1, \delta}-\delta \rho^{-1} g_{0, \delta} \frac{v_{\delta}}{2}=\mu_{0, \delta} \nabla c_{\delta}
$$

and its weak formulation (5.5) is equivalent to

$$
\begin{gathered}
-\left(P_{\sigma} v_{\delta}, \partial_{t} \psi\right)_{Q}+\left(v_{\delta} \cdot \nabla v_{\delta}, \psi\right)_{Q}+\left(\rho_{\delta}^{-1} S\left(c_{\delta}, D v_{\delta}\right), \nabla \psi\right)_{Q}+\left(S\left(c_{\delta}, D v_{\delta}\right), \nabla \rho_{\delta}^{-1} \otimes \psi\right)_{Q} \\
\quad+\gamma\left(\rho_{\delta}^{-1} v_{\delta}, \psi\right)_{S}-\left(g_{1, \delta}, \operatorname{div} \psi\right)_{Q}-\frac{\delta}{2}\left(\rho_{\delta}^{-1} g_{0, \delta} v_{\delta}, \psi\right)_{Q}=\left(\mu_{0, \delta} \nabla c_{\delta}, \psi\right)_{Q}
\end{gathered}
$$

for all $\psi \in C_{0}^{\infty}\left(0, \infty ; H_{n}^{1}(\Omega) \cap L^{\infty}(\Omega)^{d}\right)$.

Moreover, we have the following estimates uniformly in $0<\delta \leq 1$.

Lemma 6.1. Let $1<p<\frac{d}{d-1}$ and let $g_{1, \delta}, G\left(v_{\delta}\right)$ be as above. Then there is a constant $C=C(R, \varepsilon, q)$ independent of $0<\delta \leq 1$ such that

$$
\left\|G\left(v_{\delta}\right)\right\|_{L^{\infty}\left(0, \infty ; H^{1}(\Omega)\right)}+\left\|G\left(v_{\delta}\right)\right\|_{L^{2}\left(0, \infty ; H^{2}(\Omega)\right)}+\left\|g_{1, \delta}\right\|_{L^{2}\left(0, \infty ; L^{p}(\Omega)\right)} \leq C .
$$

Proof. The estimate of the first two terms simply follows from

$$
\left\|G\left(v_{\delta}(t)\right)\right\|_{H^{1}(\Omega)} \leq C\|v(t)\|_{L^{2}(\Omega)}, \quad\left\|G\left(v_{\delta}(t)\right)\right\|_{H^{2}(\Omega)} \leq C\|v(t)\|_{H^{1}(\Omega)},
$$

cf. (4.4) and (4.5). In order to estimate $g_{1, \delta}$, we choose $\varphi=\eta(t) \nabla \psi, \psi \in W_{p^{\prime}, N}^{2}(\Omega)=$ $\left\{u \in W_{p^{\prime}}^{2}(\Omega):\left.\partial_{n} u\right|_{\partial \Omega}=0\right\}, \eta \in C_{0}^{\infty}(0, \infty)$ in (6.2) and obtain

$$
\begin{aligned}
\int_{0}^{\infty} & \int_{\Omega} g_{1, \delta} \Delta \psi d x \eta(t) d t \\
= & \int_{0}^{\infty} \int_{\Omega}\left(\rho_{\delta}^{-1} S\left(c_{\delta}, D v_{\delta}\right)-v \otimes v+\frac{|v|^{2}}{2} I\right): \nabla^{2} \psi d x \eta d t \\
& +\int_{0}^{\infty} \int_{\Omega}\left(S\left(c_{\delta}, D v_{\delta}\right) \cdot \nabla \rho_{\delta}^{-1}-\mu_{0, \delta} \nabla c_{\delta}-\delta \rho^{-1} g_{0, \delta} \frac{v_{\delta}}{2}\right) \cdot \nabla \psi d x \eta d t
\end{aligned}
$$


for all $\psi \in W_{p^{\prime}, N}^{2}(\Omega), \eta \in C_{0}^{\infty}(0, \infty)$. Here we have used the identity $v$. $\nabla v=\operatorname{div}(v \otimes v)-\nabla \frac{|v|^{2}}{2}$. Hence $g_{1, \delta}(t)$ is a very weak solution of the NeumannLaplace equation, cf. Sect. 4 , for almost every $0<t<\infty$ with right-hand side $F_{\delta}(t)$ satisfying

$$
\begin{aligned}
\left\|F_{\delta}(t)\right\|_{\left(W_{p^{\prime}, N}^{2}(\Omega)\right)^{\prime}} & \\
\leq & C\left(\left\|S\left(c_{\delta}(t), D v_{\delta}(t)\right)\right\|_{L^{2}}+\|v(t)\|_{L^{s}}^{2}\right. \\
& \left.+\left\|S\left(c_{\delta}, D v_{\delta}\right) \cdot \nabla \rho_{\delta}^{-1}(t)-\mu_{0, \delta} \nabla c_{\delta}(t)-\delta \rho^{-1} g_{0, \delta} \frac{v_{\delta}}{2}\right\|_{L^{1}}\right) \\
\leq & C(R, q)\left(\left\|v_{\delta}(t)\right\|_{H^{1}(\Omega)}+\left\|\nabla \mu_{0, \delta}(t)\right\|_{L^{2}(\Omega)}\right) \in L^{2}(0, \infty),
\end{aligned}
$$

where we have used that to $W_{p^{\prime}}^{1}(\Omega) \hookrightarrow L^{\infty}(\Omega)$ as well as $\|v\|_{L^{s}}^{2} \leq\|v\|_{H^{\frac{1}{2}}}^{2} \leq$ $C\|v\|_{H^{1}}\|v\|_{L^{2}}$ with $s=3$ if $d=3$ and $s=4$ if $d=2$. Therefore

$$
\left\|g_{1, \delta}\right\|_{L^{2}\left(0, \infty ; L^{p}(\Omega)\right)} \leq C(R, \varepsilon, q)\left\|F_{\delta}(t)\right\|_{L^{2}\left(0, \infty ;\left(W_{p^{\prime}, N}^{2}(\Omega)\right)^{\prime}\right)} \leq C^{\prime}(R, \varepsilon, q)
$$

uniformly in $0<\delta \leq 1$.

Next we estimate $\bar{p}_{\delta}(t)$.

Lemma 6.2. There is a constant $C=C(R, \varepsilon, q)$ such that

$$
\left\|\bar{p}_{\delta}\right\|_{L_{\text {uloc }}^{2}([0, \infty))} \leq C \quad \text { uniformly in } \delta>0 \text {. }
$$

Proof. Choosing $\varphi=\eta(t) a^{\frac{1}{q}}\left(c_{\delta}\right) \rho_{\delta}^{-2}, \eta \in C_{0}^{\infty}(0, \infty)$ in $(5.8)$ and using $\int_{\Omega} g_{0, \delta} d x=0$, we obtain

$$
\bar{p}_{\delta}(t)=\frac{1}{|\Omega|} \int_{\Omega}\left(\rho_{\delta}^{-2} \phi\left(c_{\delta}\right)-\rho^{-1} \mu_{0, \delta}\right) d x+\frac{1}{|\Omega|} \int_{\Omega} f\left(c_{\delta}\right)\left|\nabla A\left(c_{\delta}\right)\right|^{q} d x
$$

for almost all $t \in(0, \infty)$, where $f(s)=a^{-\frac{1}{q}}(s) \frac{d}{d s}\left(a^{\frac{1}{q}}(s) \rho^{-2}(s)\right)$. Hence

$$
\left\|\bar{p}_{\delta}\right\|_{L_{\mathrm{uloc}}^{2}([0, \infty))} \leq C
$$

uniformly in $0<\delta \leq 1$ since $c_{\delta} \in L^{\infty}\left(0, \infty ; W_{q}^{1}(\Omega)\right)$ and $\mu_{0, \delta} \in L^{2}(Q)$ are uniformly bounded and $W_{q}^{1}(\Omega) \hookrightarrow L^{\infty}(\Omega)$.

Using these bounds, we obtain the following essential compactness result:

Lemma 6.3. There is a subsequence, again denoted by $\left(v_{\delta}, g_{0, \delta}, c_{\delta}, \mu_{0, \delta}, \bar{p}_{\delta}\right)_{\delta>0}$, such that

$$
\left(v_{\delta}, G\left(v_{\delta}\right)\right) \rightarrow_{\delta \rightarrow 0}(v, G(v)) \quad \text { in } L^{2}\left(0, T ; L^{2}(\Omega) \times H^{1}(\Omega)\right)
$$

for all $0<T<\infty$. 
Proof. Choosing $\varphi=a^{\frac{1}{q}}\left(c_{\delta}\right) \rho_{\delta}^{-2} \psi, \psi \in C_{0}^{\infty}(Q)$, in (5.8), we obtain

$$
-\beta \partial_{t} G\left(v_{\delta}\right)=\rho_{\delta}^{-1} \mu_{0, \delta}+\bar{p}_{\delta}(t)-\phi\left(c_{\delta}\right)-g_{1, \delta}+\rho_{\delta}^{-2} a^{\frac{1}{q}}\left(c_{\delta}\right) \Delta_{q} A\left(c_{\delta}\right) \text { in } \mathcal{D}^{\prime}(Q),
$$

where

$$
\begin{aligned}
& \left\langle\rho_{\delta}^{-2} a^{\frac{1}{q}}\left(c_{\delta}\right) \Delta_{q} A\left(c_{\delta}\right), \varphi\right\rangle_{\mathcal{D}^{\prime}(Q), \mathcal{D}(Q)} \\
& =-\int_{\Omega} \rho_{\delta}^{-2} \frac{\partial A}{\partial c}\left(c_{\delta}\right)\left|\nabla A\left(c_{\delta}\right)\right|^{q-2} \nabla A\left(c_{\delta}\right) \cdot \nabla \varphi d x+\int_{\Omega} f\left(c_{\delta}\right)\left|\nabla A\left(c_{\delta}\right)\right|^{q} \varphi d x
\end{aligned}
$$

for all $\varphi \in C_{0}^{\infty}(Q)$ and $f(s)=a^{-\frac{1}{q}}(s) \frac{d}{d s}\left(a^{\frac{1}{q}}(s) \rho^{-2}(s)\right)$ as before. Hence the last term in (6.3) is uniformly bounded in $L^{\infty}\left(0, \infty ; H^{-s}(\Omega)\right)$ if $s>\frac{d}{2}+1$, and therefore

$$
\beta \partial_{t} G\left(v_{\delta}\right) \in L_{\mathrm{uloc}}^{2}\left([0, \infty) ; H^{-s}(\Omega)\right)
$$

is uniformly bounded due to Lemma 6.1, Lemma 6.2 and since $\mu_{0, \delta} \in L^{2}(Q)$ and $\phi\left(c_{\delta}\right) \in L^{\infty}(Q)$ are bounded. On the other hand by (6.2) and Lemma 6.1,

$$
\partial_{t} P_{\sigma} v_{\delta} \in L_{\mathrm{uloc}}^{2}\left([0, \infty) ; H^{-s}(\Omega)\right) \quad \text { for } s>\frac{d}{2},
$$

see also proof of Lemma 6.1. Therefore

$$
\partial_{t} v_{\delta}=\partial_{t} P_{\sigma} v_{\delta}+\partial_{t} \nabla G\left(v_{\delta}\right) \in L_{\mathrm{uloc}}^{2}\left([0, \infty) ; H^{-s-1}(\Omega)\right) \text { for } s>\frac{d}{2}+1
$$

is uniformly bounded. Hence $v_{\delta} \rightarrow_{\delta \rightarrow 0} v$ in $L^{2}\left(Q_{T}\right)$ for every $T<\infty$ by the AubinLions lemma. Since $G\left(v_{\delta}(t)\right) \in H^{1}(\Omega)$ depends continuously on $v_{\delta}(t) \in L^{2}(\Omega)$, we obtain the second part.

As an important convergence result we also need:

Lemma 6.4. Let $F \in C^{1}(\mathbb{R})$. Then

$$
\left(G\left(v_{\delta}\right), \partial_{t}\left(F\left(c_{\delta}\right) \varphi\right)\right)_{Q} \rightarrow_{\delta \rightarrow 0}\left(G(v), \partial_{t}(F(c) \varphi)\right)_{Q}
$$

for all $\varphi \in C^{1}(\bar{Q})$ and a suitable subsequence.

Proof. First of all

$$
\partial_{t}\left(F\left(c_{\delta}\right) \varphi\right)=F^{\prime}\left(c_{\delta}\right) \partial_{t} c_{\delta} \varphi+F\left(c_{\delta}\right) \partial_{t} \varphi .
$$

Since $c_{\delta} \rightarrow_{\delta} c$ almost everywhere, $c_{\delta} \in L^{\infty}(Q)$, and $\partial_{t} c_{\delta}=-\beta^{-1} \rho^{-2} \operatorname{div}\left(\rho_{\delta} v_{\delta}\right) \in$ $L^{2}(Q)$ is uniformly bounded, cf. (4.2), we obtain

$$
F^{\prime}\left(c_{\delta}\right) \partial_{t} c_{\delta} \varphi+F\left(c_{\delta}\right) \partial_{t} \varphi \rightarrow_{\delta \rightarrow 0} F^{\prime}(c) \partial_{t} c \varphi+F(c) \partial_{t} \varphi=\partial_{t}(F(c) \varphi) \text { in } L^{2}(Q)
$$

for all $C^{1}(\bar{Q})$. Moreover, due to Lemma $6.3, G\left(v_{\delta}\right) \rightarrow_{\delta \rightarrow 0} G(v)$ strongly in $L^{2}\left(Q_{T}\right)$ for every $T<\infty$. Thus the statement of the lemma follows.

As a corollary we obtain strong convergence of $\nabla c_{\delta}$ in $L^{q}\left(Q_{T}\right)$ : 
Corollary 6.5. There is a subsequence, again denoted by $\left(c_{\delta}\right)_{0<\delta \leq 1}$, such that $\nabla c_{\delta} \rightarrow \delta \rightarrow 0$ $\nabla c$ in $L^{q}\left(Q_{T}\right)$ for every $T<\infty$.

Proof. By (5.8) we have

$$
\int_{0}^{\infty}\left\langle-\Delta_{q} A\left(c_{\delta}(t)\right), \varphi\right\rangle_{W_{q^{\prime}, 0}^{-1}, W_{q}^{1}} \eta(t) d t=-\left(G\left(v_{\delta}\right), \partial_{t}\left(F\left(c_{\delta}\right) \varphi \eta\right)\right)_{Q}+\left(f_{\delta}, \varphi \eta\right)_{Q}
$$

for all $\varphi \in C_{(0)}^{\infty}(\bar{\Omega}), \eta \in C_{0}^{\infty}(0, \infty)$, where $F\left(c_{\delta}\right)=\beta \hat{\rho}^{2}\left(c_{\delta}\right) a^{-\frac{1}{q}}\left(c_{\delta}\right)$ and

$$
f_{\delta}=a^{-\frac{1}{q}}\left(c_{\delta}\right)\left(\rho_{\delta} \mu_{0, \delta}+\rho_{\delta}^{2} \bar{p}_{\delta}(t)-\beta \rho_{\delta}^{2} g_{1, \delta}-\phi\left(c_{\delta}\right)\right) .
$$

By the convergence of $c_{\delta}$ almost everywhere and the weak convergence of $\mu_{0, \delta}, \bar{p}_{\delta}$, and $g_{1, \delta}$, we obtain

$$
f_{\delta} \rightarrow_{\delta \rightarrow 0} f \quad \text { in } L^{2}\left(0, T ; L^{p}(\Omega)\right) \text { for all } 1<p<\frac{d}{d-1},
$$

for all $T<\infty$, where

$$
f=a^{-\frac{1}{q}}(c)\left(\rho \mu_{0}+\rho^{2} \bar{p}(t)-\beta \rho^{2} g_{1}-\phi(c)\right) .
$$

Hence

$$
\lim _{\delta \rightarrow 0}\left(f_{\delta}, A\left(c_{\delta}\right) \eta\right)_{Q}=(f, A(c) \eta)_{Q}
$$

for all $\eta \in C_{0}^{\infty}(0, \infty)$. On the other hand, by Lemma 6.4,

$$
\begin{gathered}
\lim _{\delta \rightarrow 0}\left(G\left(v_{\delta}\right), \partial_{t}\left(F\left(c_{\delta}\right) \varphi \eta\right)\right)_{Q}=\left(G(v), \partial_{t}(F(c) \varphi \eta)\right)_{Q}, \\
\lim _{\delta \rightarrow 0}\left(G\left(v_{\delta}\right), \partial_{t}\left(F\left(c_{\delta}\right) A\left(c_{\delta}\right) \eta\right)\right)_{Q}=\left(G(v), \partial_{t}(F(c) A(c) \eta)\right)_{Q}
\end{gathered}
$$

for all $\eta \in C_{0}^{\infty}(0, \infty)$ and $\varphi \in C_{(0)}^{\infty}(\bar{\Omega})$. Moreover, because of (6.4), $F\left(c_{\delta}\right) \partial_{t} G\left(c_{\delta}\right)$ is uniformly bounded in $L^{q^{\prime}}\left(0, T ; W_{q^{\prime}, 0}^{-1}(\Omega)\right)$ for every $0<T<\infty$, which implies

$$
F\left(c_{\delta}\right) \partial_{t} G\left(c_{\delta}\right) \rightarrow_{\delta \rightarrow 0} F(c) \partial_{t} G(c) \quad \text { in } L^{q^{\prime}}\left(0, T ; W_{q^{\prime}, 0}^{-1}(\Omega)\right)
$$

for all $0<T<\infty$. Hence

$$
-\Delta_{q} A\left(c_{\delta}\right) \rightarrow_{\delta \rightarrow 0} F(c) \partial_{t} G(c)+f \quad \text { in } L^{q^{\prime}}\left(0, T ; W_{q^{\prime}, 0}^{-1}(\Omega)\right)=: X_{T}^{\prime} .
$$

Similarly, because of $(6.4),\left(F\left(c_{\delta}\right) \partial_{t} G\left(c_{\delta}\right), A\left(c_{\delta}\right)\right)_{\Omega}$ is uniformly bounded in $L^{2}(0, T)$ for every $0<T<\infty$. Therefore we obtain from (6.5)

$$
\lim _{\delta \rightarrow 0}\left\langle F\left(c_{\delta}\right) \partial_{t} G\left(c_{\delta}\right), A\left(c_{\delta}\right)\right\rangle_{X_{T}^{\prime}, X_{T}}=\left\langle F(c) \partial_{t} G(c), A(c)\right\rangle_{X_{T}^{\prime}, X_{T}}
$$

for all $0<T<\infty$ by passing $\eta \rightarrow 1$. Thus

$$
-\lim _{\delta \rightarrow 0}\left\langle\Delta_{q} A\left(c_{\delta}\right), A\left(c_{\delta}\right)\right\rangle_{X_{T}^{\prime}, X_{T}}=\left\langle F(c) \partial_{t} G(c)+f, A(c)\right\rangle_{X_{T}^{\prime}, X_{T}}
$$


for all $0<T<\infty$. Since $-\Delta_{q}: L^{q}\left(0, T ; W_{q, 0}^{1}(\Omega)\right) \rightarrow L^{q^{\prime}}\left(0, T ; W_{q^{\prime}, 0}^{-1}(\Omega)\right)$ is maximal monotone, cf. Remark 4.6, and because of Lemma 4.4, we conclude $-\Delta_{q} A(c)=$ $F(c) \partial_{t} G(c)+f$, and therefore

$$
\begin{aligned}
\lim _{\delta \rightarrow 0}\left\|\nabla A\left(c_{\delta}\right)\right\|_{L^{q}\left(Q_{T}\right)}^{q} & =-\lim _{\delta \rightarrow 0}\left\langle\Delta_{q} A\left(c_{\delta}\right), A\left(c_{\delta}\right)\right\rangle_{X_{T}^{\prime}, X_{T}} \\
& =-\left\langle\Delta_{q} A(c), A(c)\right\rangle_{X_{T}^{\prime}, X_{T}}=\|\nabla A(c)\|_{L^{q}\left(Q_{T}\right)}^{q}
\end{aligned}
$$

for all $T<\infty$, which proves strong convergence of $\nabla A\left(c_{\delta}\right)$ in $L^{q}\left(Q_{T}\right)$. Since $\nabla c_{\delta}=a^{-\frac{1}{q}}\left(c_{\delta}\right) \nabla A\left(c_{\delta}\right)$ and $c_{\delta} \in L^{\infty}(Q)$ converges almost everywhere, this implies the strong convergence of $\nabla c_{\delta}$ in $L^{q}\left(Q_{T}\right)$.

Proof of Theorem 2.4. Using the convergence results above, one can easily show that (6.2) converges to (2.1) and that (5.6)-(5.8) converge to (2.2)-(2.4) for a suitable subsequence $\delta_{j} \rightarrow{ }_{j \rightarrow \infty} 0$. Let us only mention the following points: Note that

$$
\delta g_{0}=\delta^{\frac{1}{2}} \delta^{\frac{1}{2}} g_{0} \rightarrow_{\delta \rightarrow 0} 0 \quad \text { in } L^{2}(Q)
$$

since $\delta^{\frac{1}{2}} g_{0} \in L^{2}(Q)$ is uniformly bounded according to (5.9). Moreover, passing to the limit $\delta \rightarrow 0$ in (5.7), one first only obtains

$$
\left(P_{0} \rho c, \partial_{t} \psi\right)_{Q}+(\rho c v, \nabla \psi)_{Q}=(m(c) \nabla \mu, \nabla \psi)_{Q}
$$

for all $\psi \in C_{0}^{\infty}\left(0, \infty ; C^{1}(\bar{\Omega})\right)$. But, because of (2.2), we have

$$
0=\int_{\Omega} \partial_{t} \rho d x=-\int_{\Omega} \beta \rho^{2} \partial_{t} c d x=-\frac{\beta}{\alpha} \int_{\Omega} \partial_{t}(\rho c) d x,
$$

since $\frac{\partial \rho}{\partial c}=-\beta \rho^{2}$ and $\frac{\partial(\rho c)}{\partial c}=\rho+\frac{\partial \rho}{\partial c} c=\alpha \rho^{2}$, cf. (3.10). Hence (2.3) follows.

By the same arguments as in the proof of Theorem 5.1 one shows that $v \in B C_{w}\left([0, \infty) ; L^{2}(\Omega)\right), c \in B C_{w}\left([0, \infty) ; W_{q}^{1}(\Omega)\right)$ and $\left.(v, c)\right|_{t=0}=\left(v_{0}, c_{0}\right)$.

Finally, the energy inequality (2.5) follows from passing to the limit (5.38) using the strong convergence of $v_{\delta}(t) \in L^{2}(\Omega), c_{\delta}(t) \in W_{q}^{1}(\Omega)$ for almost all $t \in(0, \infty)$ and Lemma 4.3 as in the proof of Theorem 5.1.

Open Access This article is distributed under the terms of the Creative Commons Attribution Noncommercial License which permits any noncommercial use, distribution, and reproduction in any medium, provided the original author(s) and source are credited.

\section{References}

1. Abels, H.: On a diffuse interface model for two-phase flows of viscous, incompressible fluids with matched densities. Arch. Rat. Mech. Anal. doi:10.1007/s00205-008-0160-2, 2008

2. Abels, H.: Diffuse interface models for two-phase flows of viscous incompressible fluids. Lecture Notes, Max Planck Institute for Mathematics in the Sciences, No. 36/2007, 2007, available at http://www.mis. mpg.de/publications/other-series/In/lecturenote-3607.html, 2008

3. Abels, H., Feireisl, E.: On a diffuse interface model for a two-phase flow of compressible viscous fluids. Indiana Univ. Math. J. 57(2), 659-698 (2008)

4. Abels, H., Wilke, M.: Convergence to equilibrium for the Cahn-Hilliard equation with a logarithmic free energy. Nonlinear Anal. 67(11), 3176-3193 (2007) 
5. Anderson, D.M., McFadden, G.B., Wheeler, A.A.: Diffuse-interface methods in fluid mechanics. In: Annual review of fluid mechanics, Vol. 30, Palo Alto, CA: Annual Reviews, 1998, pp. 139-165

6. Boyer, F.: Mathematical study of multi-phase flow under shear through order parameter formulation. Asymptot. Anal. 20(2), 175-212 (1999)

7. Boyer, F.: Nonhomogeneous Cahn-Hilliard fluids. Ann. Inst. H. Poincaré Anal. Non Linéaire 18(2), 225-259 (2001)

8. Deimling, K.: Nonlinear Functional Analysis. Berlin: Springer-Verlag, 1985

9. Diestel, J., Uhl, Jr., J.J.: Vector Measures. Providence, RI: Amer. Math. Soc., 1977

10. Gurtin, M.E., Polignone, D., Viñals, J.: Two-phase binary fluids and immiscible fluids described by an order parameter. Math. Models Methods Appl. Sci. 6(6), 815-831 (1996)

11. Lions, J.-L.: Quelques méthodes de résolution des problèmes aux limites non linéaires. Paris: Dunod, 1969

12. Liu, C., Shen, J.: A phase field model for the mixture of two incompressible fluids and its approximation by a Fourier-spectral method. Phys. D 179(3-4), 211-228 (2003)

13. Lowengrub, J., Truskinovsky, L.: Quasi-incompressible Cahn-Hilliard fluids and topological transitions. R. Soc. Lond. Proc. Ser. A Math. Phys. Eng. Sci. 454(1978), 2617-2654 (1998)

14. Roubíček, T.: A generalization of the Lions-Temam compact imbedding theorem. Časopis Pěst. Mat. 115(4), 338-342 (1990)

15. Showalter, R.E.: Monotone Operators in Banach Space and Nonlinear Partial Differential Equations. Volume 49 of Mathematical Surveys and Monographs. Providence, RI: Amer. Math. Soc., 1997

16. Simon, J.: Compact sets in the space $L^{p}(0, T ; B)$. Ann. Mat. Pura Appl. (4), 146, 65-96, (1987)

17. Starovoltov, V.N.: On the motion of a two-component fluid in the presence of capillary forces. Mat. Zametki 62(2), 293-305 (1997)

18. Stein, E.M.: Singular Integrals and Differentiability Properties of Functions. Princeton, NJ: Princeton Univ. Press, 1970

19. Triebel, H.: Interpolation Theory, Function Spaces, Differential Operators. Amsterdam-New YorkOxford: North-Holland Publishing Company, 1978

Communicated by P. Constantin 